\title{
Optimal partial regularity of second-order parabolic systems under natural growth condition
}

\author{
Shuhong Chen ${ }^{1}$ and Zhong $\operatorname{Tan}^{2^{*}}$
}

"Correspondence: ztan85@163.com ${ }^{2}$ School of Mathematical Science, Xiamen University, Xiamen, Fujian 361005, China

Full list of author information is available at the end of the article

\begin{abstract}
We consider the regularity for weak solutions of second-order nonlinear parabolic systems under a natural growth condition when $m>2$, and obtain a general criterion for a weak solution to be regular in the neighborhood of a given point. In particular, we get the optimal regularity by the method of A-caloric approximation introduced by Duzaar and Mingione.
\end{abstract}

Keywords: nonlinear parabolic systems; natural growth condition; A-caloric approximation; optimal partial regularity

\section{Introduction}

Electrorheological fluids are special viscous liquids, that are characterized by their ability to undergo significant changes in their mechanical properties when an electric field is applied. This property can be exploited in technological applications, e.g., actuators, clutches, shock absorbers, and rehabilitation equipment to name a few [1].

A model was developed for these liquids within the framework of rational mechanics $[2,3]$; it takes into account the complex interactions between the electro-magnetic fields and the moving liquid. If the fluid is assumed to be incompressible, it turns out that the relevant equations of the model are the system

$$
\begin{aligned}
& \operatorname{div}(E+P)=0 \\
& \operatorname{curl} E=0 \\
& \rho_{0} \frac{\partial v}{\partial t}-\operatorname{div} S+\rho_{0}[\nabla v] v+\nabla \phi=\rho_{0} f+[\nabla E] P, \\
& \operatorname{div} v=0
\end{aligned}
$$

where $E$ is the electric field, $P$ is the polarization, $\rho_{0}$ is the density, $v$ is the velocity, $S$ is the extra stress, $\phi$ is the pressure, and $f$ is the mechanical force. In fact, in a model capable of explaining many of the observed phenomena, the extra stress has the form

$$
\begin{aligned}
S= & \alpha_{21}\left(\left(1+|D|^{2}\right)^{\frac{p-1}{2}}-1\right) E \otimes E+\left(\alpha_{31}+\alpha_{33}|E|^{2}\right)\left(1+|D|^{2}\right)^{\frac{p-2}{2}} D \\
& +\alpha_{51}\left(1+|D|^{2}\right)^{\frac{p-2}{2}}(D E \otimes E+E \otimes D E),
\end{aligned}
$$


where $\alpha_{i j}$ are material constants, and where the material function $p$ depends on the strength of the electric field $|E|^{2}$ and satisfies

$$
1<p_{\infty} \leq p\left(|E|^{2}\right) \leq p_{0}<\infty
$$

Since the material function $p$, which essentially determines $S$, depends on the magnitude of the electric field $|E|^{2}$, we have to deal with an elliptic or parabolic system of partial differential equations with the so-called non-standard growth conditions, i.e., the elliptic operator $S$ satisfies

$$
\begin{aligned}
& S(D, E) \cdot D \geq c_{0}\left(1+|E|^{2}\right)\left(1+|D|^{2}\right)^{\frac{p_{\infty}-2}{2}}|D|^{2}, \\
& |S(D, E)| \leq c_{1}\left(1+|D|^{2}\right)^{\frac{p_{0}-1}{2}}|E|^{2} .
\end{aligned}
$$

Equality (1.5) of electrorheological fluids with the conditions (1.7) and (1.8) encouraged us to considered the partial regularity of a more simple and standard model as the following:

$$
u_{t}^{i}-\sum_{\alpha=1}^{n} D_{\alpha} A_{i}^{\alpha}(z, u, D u)=B_{i}(z, u, D u), \quad i=1,2, \ldots, N
$$

where $\Omega \subset R^{n}$ is a bounded domain and $T>0, z=(x, t)$ with $x \in \Omega, 0<t \leq T$, denote a point in $Q_{T}=\Omega \times(-T, 0)$. Let $u(z)=\left(u^{1}(z), u^{2}(z), \ldots, u^{N}(z)\right)$ be a vector-valued function defined in $Q_{T}$. Denote by $D u$ the gradient of $u$, i.e., $D u=\left\{D_{\alpha} u^{i}\right\}_{i=1, \ldots, N ; \alpha=1, \ldots, n} . m>2$ is a real number.

In order to define the weak solution of (1.9), one needs to impose some regularity conditions and constructer conditions to $A_{i}^{\alpha}$ and $B_{i}$. For a vector field $A_{i}^{\alpha}: Q_{T} \times R^{N} \times R^{n N}$, we shall denote the coefficients by $A_{i}^{\alpha}(z, u, p)=A_{i}^{\alpha}(x, t, u, p)$ if $z=(x, t), u \in R^{N}$ and $p \in R^{n N}$. We assume that the functions $(z, u, p) \mapsto A_{i}^{\alpha}(z, u, p) ;(z, u, p) \mapsto \frac{\partial A_{i}^{\alpha}}{\partial p_{\beta}^{j}}(z, u, p)$ are continuous in $Q_{T} \times R^{N} \times R^{n N}$ and that the following growth and ellipticity conditions are satisfied:

(H1) There exists a constant $L$ such that

$$
\left|A_{i}^{\alpha}(z, u, p)\right| \leq L(1+|p|)^{\frac{m}{2}} \quad \text { for all } z \in Q_{T}, u \in R^{n} \text { and } p \in R^{n N} .
$$

(H2) $A_{i}^{\alpha}(z, u, p)$ are differentiable functions in $p$ and there exists a constant $L$ such that

$$
\left|\frac{\partial A_{i}^{\alpha}}{\partial p_{\beta}^{i}}(z, u, p)\right| \leq L\left(1+|p|^{2}\right)^{\frac{m-2}{2}} \quad \text { for all } z \in Q_{T}, u \in R^{n} \text { and } p \in R^{n N}
$$

(H3) $A_{i}^{\alpha}$ is uniformly strongly elliptic, that is, for some $\lambda>0$, we have

$$
\left(\frac{\partial A_{i}^{\alpha}}{\partial p_{\beta}^{i}}(z, u, p) \tilde{p}_{\alpha}^{i}\right) \cdot \tilde{p}_{\beta}^{j} \geq \lambda|\tilde{p}|^{2}\left(1+|p|^{2}\right)^{\frac{m-2}{2}} \quad \text { for all } z \in Q_{T}, u \in R^{n} \text { and } p, \tilde{p} \in R^{n N}
$$

where $\lambda>0$ and $1 \leq L<\infty$. Now we shall specify the regularity assumptions on $A_{i}^{\alpha}(z, u, p)$ with respect to the 'coefficient' $(z, u)$ and assume that the function $(z, u) \mapsto$ 
$\frac{A_{i}^{\alpha}(z, u, p)}{1+|p|}$ is Hölder continuous with respect to the parabolic metric $\sqrt{\left|x-x_{0}\right|^{2}+\left|t-t_{0}\right|}$ with Hölder exponent $\beta \in(0,1)$ but not necessarily uniformly Hölder continuous; namely we shall assume that:

(H4) There exists a constant $L$ such that

$$
\left|A_{i}^{\alpha}(z, u, p)-A_{i}^{\alpha}\left(z_{0}, u_{0}, p\right)\right| \leq L \theta\left(|u|+\left|u_{0}\right|,\left|x-x_{0}\right|+\sqrt{\left|t-t_{0}\right|}+\left|u-u_{0}\right|\right)(1+|p|)^{\frac{m}{2}}
$$

for any $z=(x, t)$ and $z_{0}=\left(x_{0}, t_{0}\right)$ in $Q_{T} . u$ and $u_{0}$ in $R^{n}$ and for all $p \in R^{n N}$, where $\theta(y, s)=\min \left\{1, \tilde{K}(y) s^{\beta}\right\}, \tilde{K}:[0, \infty) \mapsto(1, \infty)$ is a given non-decreasing function. Note that $\theta$ is concave in the argument. This is the standard way to prescribe (nonuniform) Hölder continuity of the function $A_{i}^{\alpha}(z, u, p)$. We find it a bit difficult to handle, therefore, in many points of the paper, we shall use:

$\left(\mathrm{H} 4^{\prime}\right)$ For $\beta \in(0,1)$ and $K:[0, \infty) \rightarrow[L, \infty)$ monotone nondecreasing such that

$$
\left|A_{i}^{\alpha}(z, u, p)-A_{i}^{\alpha}\left(z_{0}, u_{0}, p\right)\right| \leq K(|u|)\left(\left|x-x_{0}\right|+\sqrt{\left|t-t_{0}\right|}+\left|u-u_{0}\right|\right)^{\beta}(1+|p|)^{\frac{m}{2}},
$$

valid for any $z=(x, t)$ and $z_{0}=\left(x_{0}, t_{0}\right)$ in $Q_{T}, u$ and $u_{0}$ in $R^{n}$ and $p \in R^{n N}$.

(H5) There exist constants $a$ and $b$ such that

$$
\left|B_{i}(z, u, p)\right| \leq a|p|^{m}+b, \quad \sup _{Q_{T}}|u|=V, \quad 2 a V<\lambda .
$$

Finally, we remark a trial consequence of the continuity of $\frac{\partial A_{i}^{\alpha}}{\partial p_{\beta}^{j}}$; this implies the existence of a function $\omega:[0, \infty) \times[0, \infty) \mapsto[0, \infty)$ with $\omega(t, 0)=0$ for all $t$ such that $t \mapsto \omega(t, s)$ is nondecreasing for fixed $s, s \mapsto \omega^{m}(t, s)$ is concave and nondecreasing for fixed $t$, and such that

$$
\begin{aligned}
& \left|\frac{\partial A_{i}^{\alpha}}{\partial p_{\beta}^{j}}(x, t, u, p)-\frac{\partial A_{i}^{\alpha}}{\partial p_{\beta}^{j}}\left(x_{0}, t_{0}, u_{0}, p_{0}\right)\right| \\
& \quad \leq L\left(1+|p|^{2}+\left|p_{0}\right|^{2}\right)^{\frac{m-2}{2}} \\
& \quad \times \omega\left(|u|+|p|,\left|x-x_{0}\right|^{2}+\left|t-t_{0}\right|+\left|u-u_{0}\right|^{2}+\left|p-p_{0}\right|^{2}\right)
\end{aligned}
$$

for any $z=(x, t)$ and $z_{0}=\left(x_{0}, t_{0}\right)$ in $Q_{T}$, any $u, u_{0}$ in $R^{n}$ and $p, p_{0} \in R^{n N}$ whenever $|u|+|p|+$ $\left|u-u_{0}\right|+\left|p-p_{0}\right| \leq M$.

From $(\mathrm{H} 2)$ and $(\mathrm{H} 3)$ we immediately deduce the following:

$$
\begin{aligned}
& \left|A_{i}^{\alpha}(z, u, p)-A_{i}^{\alpha}(z, u, q)\right| \leq L\left(1+|p|^{2}+|q|^{2}\right)^{\frac{m-2}{2}}|p-q|, \\
& \left(A_{i}^{\alpha}(z, u, p)-A_{i}^{\alpha}(z, u, q)\right)(p-q) \geq \lambda\left(1+|p|^{2}+|q|^{2}\right)^{\frac{m-2}{2}}|p-q|^{2}
\end{aligned}
$$

for all $z \in Q_{T}, u \in R^{N}$ and $p, q \in R^{n N}$.

Definition 1.1 By a weak solution of (1.9) under the assumptions (H1)-(H5), we mean a vector-valued function $u \in L^{m}\left(-T, 0 ; W^{1, m}\left(\Omega, R^{N}\right)\right) \cap L^{\infty}\left(Q_{T} ; R^{N}\right)$ such that

$$
\int_{Q_{T}}\left(A_{i}^{\alpha}(z, u, D u) D_{\alpha} \varphi^{i}-u^{i} \varphi_{t}^{i}\right) d z=\int_{Q_{T}} B_{i}(z, u, D u) \cdot \varphi^{i} d z
$$

for all $\varphi \in C_{0}^{\infty}\left(Q_{T}, R^{N}\right)$. 
In [4] Duzaar and Mingione considered the partial regularity of homogeneous systems of (1.9) with $m \equiv 2$ under the natural growth condition. In this paper, we extend their results to the case of $m>2$. We have to overcome the difficulty of $m>2$. Motivated by the works of Duzaar [4, 5], Chen and Tan [6-9] and Tan [10], we use the technique of 'A-caloric approximation' to establish the optimal partial regularity of nonlinear parabolic systems (1.9). In fact, the use of the ' $A$-caloric approximation lemma' allows optimal regularity, without the use of Reverse-Hölder inequalities and (parabolic) Gehring's lemma. The method is based on an approximation result that we called the ' $A$-caloric approximation lemma.' This is the parabolic analogue of the classical harmonic approximation lemma of De Giorgi [11,12] and allows to approximate functions with solutions to parabolic systems with constant coefficients in a similar way as the classical harmonic approximation lemma does with harmonic functions. And we can obtain the following theorem.

Theorem 1.1 Let $u \in L^{m}\left(-T, 0 ; W^{1, m}\left(\Omega, R^{N}\right)\right) \cap L^{\infty}\left(Q_{T} ; R^{N}\right)$ be a weak solution to system (1.9) under the assumptions $(\mathrm{H} 1)-(\mathrm{H} 4)$ and the natural growth condition $(\mathrm{H} 5)$ and denote by $Q_{0}$ the set of regularity points of $u$ in $Q_{T}$ :

$$
Q_{0}=\left\{z \in Q_{T}: D u \in C^{\beta, \beta / 2}\left(O, R^{n N}\right), O \subset Q_{T} \text { is a neighborhood of } z\right\} .
$$

Then $Q_{0}$ is an open subset with full measure, and therefore

$$
D u \in C^{\beta, \beta / 2}\left(Q_{0}, R^{n N}\right), \quad\left|Q_{T} \backslash Q_{0}\right|=0 .
$$

At the end of the section, we summarize some notions which we will be used in this paper. For $x_{0} \in R^{n}, t_{0} \in R$, we denote $B\left(x_{0}, R\right)=\left\{x \in R^{n}:\left|x-x_{0}\right|<R\right\}, Q\left(\left(x_{0}, t_{0}\right), R\right)=B\left(x_{0}, R\right) \times$ $\left(t_{0}-R^{2}, t_{0}\right)$. If $v$ is an integrable function in $Q\left(z_{0}, \rho\right)=Q_{\rho}\left(z_{0}\right)=B_{\rho}\left(x_{0}\right) \times\left(t_{0}-\rho^{2}, t_{0}\right)$, $z_{0}=\left(x_{0}, t_{0}\right)$, we will denote its average by $(v)_{z_{0}, \rho}=f_{Q_{\rho}\left(z_{0}\right)} v d z=\frac{1}{\alpha_{n} \rho^{n+2}} \int_{Q_{\rho}\left(z_{0}\right)} v d z$, where $\alpha_{n}$ denotes the volume of the unit ball in $R^{n}$. We remark that in the following, when not crucial, the 'center' of the cylinder will be often unspecified, e.g., $Q_{\rho}\left(z_{0}\right)=Q_{\rho}$; the same convention will be adopted for balls in $R^{n}$ therefore denoting $B\left(x_{0}, \rho\right)=B_{\rho}\left(x_{0}\right)$. Finally, in the rest of the paper, the symbol $C$ will denote a positive, finite constant that may vary from line to line; the relevant dependencies will be specified.

\section{The $A$-caloric approximation technique and preliminaries}

In this section we introduce the $A$-caloric approximation lemma [4] and some preliminaries. Recall a strongly elliptic bilinear form $A_{i}^{\alpha}$ on $R^{n N}$ with an ellipticity constant $\lambda>0$, and upper bound $\Lambda>0$ means that $\lambda|\tilde{p}|^{2} \leq A_{i}^{\alpha}(\tilde{p}, \tilde{p}), A_{i}^{\alpha}(p, \tilde{p}) \leq \Lambda|p \| \tilde{p}|, \forall p, \tilde{p} \in R^{n N}$, we define $A$-caloric approximation function.

Definition 2.1 We shall say that a function $h \in L^{2}\left(-1,0 ; W^{1,2}\left(B_{\rho}, R^{N}\right)\right)$ is $A$-caloric on $Q_{\rho}$ if it satisfies

$$
\int_{Q_{\rho}}\left(h^{i} \varphi_{t}^{i}-A_{i}^{\alpha}\left(D h, D_{\alpha} \varphi^{i}\right)\right) d z=0 \quad \text { for all } \varphi \in C_{0}^{\infty}\left(Q_{\rho}, R^{N}\right) .
$$

Remark 2.2 Obviously, when $A(\tilde{p}, \tilde{p}) \equiv|\tilde{p}|^{2}$ for every $\tilde{p} \in R^{n N}$, then an $A$-caloric function is just a caloric function $h_{t}-\Delta h \equiv 0$. 
Lemma 2.3 (A-caloric approximation lemma) There exists a positive function $\delta(n, N, \lambda, \Lambda$, $\varepsilon) \leq 1$ with the following property: Whenever $A$ is a bilinear form on $R^{n N}$, which is strongly ellipticity constant $\lambda>0$ and upper bound $\Lambda, \varepsilon$ is a positive number, and $u \in$ $L^{2}\left(-1,0 ; W^{1,2}\left(B, R^{N}\right)\right)$ with

$$
\int_{Q}\left(|u|^{2}+|D u|^{2}\right) d z \leq 1
$$

is approximatively A-caloric in the sense that

$$
\left|\int_{Q}\left(u \varphi_{t}-A(D u, D \varphi)\right) d z\right| \leq \delta \sup _{Q}|D \varphi| \quad \text { for all } \varphi \in C_{0}^{\infty}\left(Q, R^{N}\right)
$$

then there exists an A-caloric function $h$ such that

$$
\int_{Q}\left(|h|^{2}+|D h|^{2}\right) d z \leq 1, \text { and } \int_{Q}|u-h|^{2} d z \leq \varepsilon .
$$

Actually, we could have directly applied Theorem 5 of [13] with the choice $X=$ $W^{1,2}\left(B, R^{N}\right), B=L^{2}\left(B, R^{N}\right), R=W^{-l, 2}\left(B, R^{N}\right), F=\left(v_{k}\right)_{k \in N}, p=2$ to conclude that $\left(v_{k}\right)_{k \in N}$ is relatively compact in $L^{2}\left(Q_{T}, R^{N}\right)=L^{2}\left(-1,0 ; L^{2}\left(B, R^{N}\right)\right)$.

Lemma 2.4 There exists a positive function $\delta(n, N, \lambda, \Lambda, \varepsilon) \leq 1$ with the following property: Whenever $A$ is a bilinear form on $R^{n N}$ which is strongly ellipticity constant $\lambda>0$ and upper bound $\Lambda, \varepsilon$ is a positive number, and $u \in L^{2}\left(t_{0}-\rho^{2}, t_{0} ; W^{1,2}\left(B_{\rho}\left(x_{0}\right), R^{N}\right)\right)$ with

$$
\rho^{-2} f_{Q_{\rho}\left(z_{0}\right)}|u|^{2} d z+f_{Q_{\rho}\left(z_{0}\right)}|D u|^{2} d z \leq 1
$$

is approximatively A-caloric in the sense that

$$
\left|f_{Q_{\rho}\left(z_{0}\right)}\left(u \varphi_{t}-A(D u, D \varphi)\right) d z\right| \leq \delta \sup _{Q_{\rho}\left(z_{0}\right)}|D \varphi| \quad \text { for all } \varphi \in C_{0}^{\infty}\left(Q_{\rho}\left(z_{0}\right), R^{N}\right)
$$

then there exists $h \in L^{2}\left(t_{0}-\rho^{2}, t_{0} ; W^{1,2}\left(B_{\rho}\left(x_{0}\right), R^{N}\right)\right) A$-caloric on $Q_{\rho}\left(z_{0}\right)$ such that

$$
\rho^{-2} f_{Q_{\rho}\left(z_{0}\right)}|h|^{2} d z+f_{Q_{\rho}\left(z_{0}\right)}|D h|^{2} d z \leq 1, \quad \text { and } \quad \rho^{-2} f_{Q_{\rho}\left(z_{0}\right)}|u-h|^{2} d z \leq \varepsilon
$$

For $u \in L^{2}\left(Q_{\rho}\left(z_{0}\right), R^{N}\right)$ we denote by $l_{z_{0}, \rho}$ the unique affine function (in space) $l(z)=l(x)$ minimizing $l \mapsto f_{Q_{\rho}\left(z_{0}\right)}|u-l|^{2} d z$, amongst all affine functions $a(z)=a(x)$ which are independent of $t$. To get an explicit formula for $l_{z_{0}, \rho}$, we note that such a unique minimum point exists and takes the form $l_{z_{0}, \rho}(x)=\xi_{z_{0}, \rho}+v_{z_{0}, \rho}\left(x-x_{0}\right)$, where $v_{z_{0}, \rho} \in R^{n N}$. A straightforward computation yields that $f_{Q_{\rho}\left(z_{0}\right)} u \cdot a(x) d z=f_{Q_{\rho}\left(z_{0}\right)} l_{z_{0}, \rho}(x) \cdot a(x) d z$, for any affine function $a(x)=\xi+v\left(x-x_{0}\right)$ with $\xi \in R^{N}$ and $v \in R^{n N}$. This implies in particular that $\xi_{z_{0}, \rho}=f_{Q_{\rho}\left(z_{0}\right)} u d z=(u)_{z_{0}, \rho}$ and $v_{z_{0}, \rho}=\frac{n+2}{\rho^{2}} f_{Q_{\rho}\left(z_{0}\right)} u \otimes\left(x-x_{0}\right) d z$.

For convenience we recall from [14] the following. 
Lemma 2.5 Let $u \in L^{2}\left(Q_{\rho}\left(z_{0}\right), R^{N}\right), 0<\theta<1$, and $l_{z_{0}, \rho}$ respectively $l_{z_{0}, \theta \rho}$ the unique affine functions minimizing $l \mapsto f_{Q_{\rho}\left(z_{0}\right)}|u-l|^{2} d z$ respectively $l \mapsto f_{Q_{\theta \rho}\left(z_{0}\right)}|u-l|^{2} d z$. Then there holds

$$
\left|v_{z_{0}, \theta \rho}-v_{z_{0}, \rho}\right|^{2} \leq \frac{n(n+2)}{(\theta \rho)^{2}} f_{Q_{\theta \rho}\left(z_{0}\right)}\left|u-(u)_{z_{0}, \rho}-v_{z_{0}, \rho}\left(x-x_{0}\right)\right|^{2} d z .
$$

Moreover, if $D u \in L^{2}\left(Q_{\rho}\left(z_{0}\right), R^{n N}\right)$, we have

$$
\left|v_{z_{0}, \rho}-(D u)_{z_{0}, \rho}\right|^{2} \leq \frac{n(n+2)}{\rho^{2}} f_{Q_{\rho}\left(z_{0}\right)}\left|u-(u)_{z_{0}, \rho}-(D u)_{z_{0}, \rho}\left(x-x_{0}\right)\right|^{2} d z .
$$

\section{Caccioppoli second inequality}

In this section we prove Caccioppoli's second inequality.

Theorem 3.1 (Caccioppoli second inequality) Let $u \in L^{m}\left(-T, 0 ; W^{1, m}\left(\Omega, R^{N}\right)\right) \cap L^{\infty}\left(Q_{T}\right.$; $\left.R^{N}\right)$ be a weak solution to (1.9) under the assumptions $(\mathrm{H1})-(\mathrm{H} 4)$ and the natural growth condition (H5). Then, for any $M>0$, any affine function $l(z)=l(x)$ independent of $t$ and satisfying $\left|l\left(z_{0}\right)\right|+|D l| \leq M$, and any $Q_{\rho}\left(z_{0}\right) \subset \subset Q_{T}$ with $0<\rho<R \leq 1$, we have

$$
\begin{aligned}
& f_{Q_{\rho}\left(z_{0}\right)}\left[\left(1+|D l|^{2}\right)^{\frac{m-2}{2}}|D u-D l|^{2}+|D u-D l|^{m}\right] d z \\
& \quad \leq C_{\mathrm{cac}}\left\{\left(1+|D l|^{2}\right)^{\frac{m-2}{2}} f_{Q_{R}\left(z_{0}\right)} \frac{1}{(R-\rho)^{2}}|u-l|^{2} d z+f_{Q_{R}\left(z_{0}\right)} \frac{1}{(R-\rho)^{m}}|u-l|^{m} d z\right. \\
& \left.\quad+\left[K(|l|)(1+|D l|)^{\frac{m}{2}}\right]^{\frac{2}{1-\beta}} R^{2 \beta}+\left(b^{2}+a^{2}|D l|^{2 m}\right) R^{2}\right\} .
\end{aligned}
$$

Proof We take the test function $\varphi=\eta^{2} \xi^{2}(u-l)$, where $\eta(x) \in C_{0}^{1}\left(B_{R}\left(x_{0}\right)\right)$ is a cut-off function in space such that $0 \leq \eta \leq 1, \eta \equiv 1$ in $B_{\rho}\left(x_{0}\right),|D \eta| \leq \frac{1}{(R-\rho)}$. While $\xi \in C^{1}(R)$ is a cut-off function in time such that, with $0<\sigma<\rho$ being arbitrary,

$$
\begin{cases}\xi \equiv 1, & \text { on }\left(t_{0}-\rho^{2}, t_{0}-\sigma^{2}\right), \\ \xi \equiv 0, & \text { on }\left(-\infty, t_{0}-R^{2}\right) \cup\left(t_{0}, \infty\right) \\ 0 \leq \xi \leq 1, & \text { on } R, \\ \xi_{t} \leq 0, & \text { on }\left(t_{0}-\rho^{2}, \infty\right) \\ \left|\xi_{t}\right| \leq \frac{1}{|R-\rho|^{2}}, & \text { on }\left(t_{0}-R^{2}, t_{0}-\rho^{2}\right) .\end{cases}
$$

Thus, we obtain

$$
\begin{aligned}
& \int_{Q_{R}\left(z_{0}\right)} A_{i}^{\alpha}(z, u, D u) D(u-l)^{i} \xi^{2} \eta^{2} d z \\
& =-2 \int_{Q_{R}\left(z_{0}\right)} A_{i}^{\alpha}(z, u, D u) \xi^{2} \eta \nabla \eta \otimes(u-l)^{i} d z \\
& \quad+\int_{Q_{R}\left(z_{0}\right)} u^{i} \partial_{t} \varphi^{i} d z+\int_{Q_{R}\left(z_{0}\right)} B_{i}(z, u, D u) \varphi^{i} d z .
\end{aligned}
$$


We further have

$$
\begin{aligned}
& -\int_{Q_{R}\left(z_{0}\right)} A_{i}^{\alpha}(z, u, D l) D_{\alpha}(u-l)^{i} \xi^{2} \eta^{2} d z \\
& \quad=2 \int_{Q_{R}\left(z_{0}\right)} A_{i}^{\alpha}(z, u, D l) \xi^{2} \eta \nabla \eta \otimes(u-l)^{i} d z-\int_{Q_{R}\left(z_{0}\right)} A_{i}^{\alpha}(z, u, D l) D_{\alpha} \varphi^{i} d z
\end{aligned}
$$

and

$$
0=\int_{Q_{R}\left(z_{0}\right)} A_{i}^{\alpha}\left(z_{0}, l\left(z_{0}\right), D l\right) D_{\alpha} \varphi^{i} d z
$$

Adding these equations and using $l_{t} \equiv 0$, we deduce

$$
\begin{aligned}
\int_{Q_{R}\left(z_{0}\right)} & {\left[A_{i}^{\alpha}(z, u, D u)-A_{i}^{\alpha}(z, u, D l)\right] D(u-l) \xi^{2} \eta^{2} d z } \\
= & -2 \int_{Q_{R}\left(z_{0}\right)}\left[A_{i}^{\alpha}(z, u, D u)-A_{i}^{\alpha}(z, u, D l)\right] \xi^{2} \eta \nabla \eta \otimes(u-l) d z \\
& -\int_{Q_{R}\left(z_{0}\right)}\left[A_{i}^{\alpha}(z, u, D l)-A_{i}^{\alpha}(z, l, D l)\right] D_{\alpha} \varphi^{i} d z \\
& -\int_{Q_{R}\left(z_{0}\right)}\left[A_{i}^{\alpha}(z, l, D l)-A_{i}^{\alpha}\left(z_{0}, l\left(z_{0}\right), D l\right)\right] D_{\alpha} \varphi^{i} d z \\
& +\int_{Q_{R}\left(z_{0}\right)}(u-l)^{i} \partial_{t} \varphi^{i} d z+\int_{Q_{R}\left(z_{0}\right)} B_{i}(z, u, D u) \varphi^{i} d z \\
\leq & I+I I+I I I+I V+V .
\end{aligned}
$$

By (1.10) and Young's inequality, we have

$$
\begin{aligned}
I \leq & \varepsilon \int_{Q_{R}\left(z_{0}\right)}\left(1+|D l|^{2}\right)^{\frac{m-2}{2}}|D u-D l|^{2} \xi^{2} \eta^{2} d z+\varepsilon^{-(m-1)} C^{m} \int_{Q_{R}\left(z_{0}\right)} \xi^{m}|\nabla \eta|^{m}|u-l|^{m} d z \\
& +\frac{C^{2}}{\varepsilon} \int_{Q_{R}\left(z_{0}\right)}\left(1+|D l|^{2}\right)^{\frac{m-2}{2}} \xi^{2}|\nabla \eta|^{2}|u-l|^{2} d z \\
& +\varepsilon \int_{Q_{R}\left(z_{0}\right)}|D u-D l|^{m} \xi^{\frac{m}{m-1}} \eta^{\frac{m}{m-1}} d z
\end{aligned}
$$

By the condition ( $\left.\mathrm{H}^{\prime}\right)$ and Young's inequality, we can get

$$
\begin{aligned}
I I \leq & \varepsilon \int_{Q_{R}\left(z_{0}\right)} \xi^{2} \eta^{2}|D u-D l|^{2} d z+\left(\frac{1}{\varepsilon}+1\right) \frac{1}{|R-\rho|^{2}} \int_{Q_{R}\left(z_{0}\right)} \xi^{2} \eta|u-l|^{2} d z \\
& +\left(\frac{1}{\varepsilon}+2^{\frac{2}{1-\beta}}\right)\left[K(|l|)(1+|D l|)^{\frac{m}{2}}\right]^{\frac{2}{1-\beta}} \alpha_{n} R^{n+2+\frac{2 \beta}{1-\beta}} .
\end{aligned}
$$

Similarly, we can estimate $I I I$ as follows:

$$
\begin{aligned}
I I I \leq & \varepsilon \int_{Q_{R}\left(z_{0}\right)} \xi^{2} \eta^{2}|D u-D l|^{2} d z+\int_{Q_{R}\left(z_{0}\right)} \xi^{2}|\nabla \eta|^{2}|u-l|^{2} d z \\
& +\left(\frac{1}{\varepsilon}+4\right)\left[K(|l|)(1+|D l|)^{\frac{m}{2}+\beta}\right]^{2} \alpha_{n} R^{n+2+2 \beta} .
\end{aligned}
$$


Using the fact that $\xi \equiv 0$ on $\left(-\infty, t_{0}-R^{2}\right) \cup\left(t_{0}, \infty\right)$, taking into account that $\xi \xi_{t} \leq 0$ for $t>t_{0}-\rho^{2}$ and $\left|\xi_{t}\right| \leq \frac{1}{|R-\rho|^{2}}$, we infer

$$
\begin{aligned}
I V & =\int_{Q_{R}\left(z_{0}\right)}(u-l)^{i} \partial_{t} \varphi^{i} d z=\int_{Q_{R}\left(z_{0}\right)}|u-l|^{2} \eta^{2} \partial_{t}\left(\xi^{2}\right) d z+\frac{1}{2} \int_{Q_{R}\left(z_{0}\right)} \xi^{2} \eta^{2} \partial_{t}|u-l|^{2} d z \\
& =\frac{1}{2} \int_{Q_{R}\left(z_{0}\right)}|u-l|^{2} \eta^{2} \partial_{t}\left(\xi^{2}\right) d z=\int_{Q_{R}\left(z_{0}\right)}|u-l|^{2} \eta^{2} \xi \xi_{t} d z \\
& \leq \frac{1}{|R-\rho|^{2}} \int_{Q_{R}\left(z_{0}\right)}|u-l|^{2} d z
\end{aligned}
$$

and for $\mu$ positive to be fixed later, we have

$$
\begin{aligned}
V= & \int_{Q_{R}\left(z_{0}\right)} a|D u|^{m} \xi^{2} \eta^{2}|u-l| d z+\int_{Q_{R}\left(z_{0}\right)}\left(\frac{|u-l|}{R-\rho} \xi \eta\right)(\xi \eta b(R-\rho)) d z \\
\leq & \int_{Q_{R}\left(z_{0}\right)} a\left[(1+\mu)|D u-D l|^{m}+\left(1+\frac{1}{\mu}\right)|D l|^{m}\right] \xi^{2} \eta^{2}|u-l| d z \\
& +\frac{1}{2 \varepsilon} \int_{Q_{R}\left(z_{0}\right)}\left(\frac{|u-l|}{R-\rho} \xi \eta\right)^{2} d z+\frac{\varepsilon}{2} \int_{Q_{R}\left(z_{0}\right)} \xi^{2} \eta^{2} b^{2} R^{2} d z \\
\leq & a V(1+\mu) \int_{Q_{R}\left(z_{0}\right)} \xi^{2} \eta^{2}|D u-D l|^{m} d z+\frac{1}{\varepsilon} \int_{Q_{R}\left(z_{0}\right)}\left(\frac{|u-l|}{R-\rho} \xi \eta\right)^{2} d z \\
& +\frac{\varepsilon}{2}\left[a^{2}\left(1+\frac{1}{\mu}\right)^{2}|D l|^{2 m}+b^{2}\right] \alpha_{n} R^{n+4} .
\end{aligned}
$$

By (1.11) we have

$$
\begin{aligned}
& \int_{Q_{R}\left(z_{0}\right)}\left[A_{i}^{\alpha}(z, u, D u)-A_{i}^{\alpha}(z, u, D l)\right] D_{\alpha}(u-l)^{i} \xi^{2} \eta^{2} d z \\
& \quad \geq \lambda \int_{Q_{R}\left(z_{0}\right)}\left(1+|D u|^{2}+|D l|^{2}\right)^{\frac{m-2}{2}}|D u-D l|^{2} \xi^{2} \eta^{2} d z \\
& \quad \geq \lambda \int_{Q_{R}\left(z_{0}\right)}\left[\left(1+|D l|^{2}\right)^{\frac{m-2}{2}}|D u-D l|^{2} \xi^{2} \eta^{2}+|D u-D l|^{m} \xi^{2} \eta^{2}\right] d z .
\end{aligned}
$$

Combining (3.2)-(3.7) in (3.1) and noting that $R^{\frac{2 \beta}{1-\beta}} \leq R^{2 \beta}(R \leq 1)$, that $2^{\frac{2}{1-\beta}}>4$, that $\left[K(|l|)(1+|D l|)^{\frac{m}{2}+\beta}\right]^{2} \leq\left[K(|l|)(1+|D l|)^{\frac{m}{2}}\right]^{\frac{2}{1-\beta}}$ (for $\left.K \geq 1\right)$, choosing $\varepsilon$ sufficiently small and taking into account that $2 a V \leq \lambda$, that $\xi \equiv 1$ for $t \in\left[t_{0}-\rho^{2}, t_{0}-\sigma^{2}\right]$, that $\eta \equiv 1$ on $B_{\rho}\left(x_{0}\right)$, we infer that

$$
\begin{aligned}
& \int_{t_{0}-\rho^{2}}^{t_{0}-\sigma^{2}} \int_{B_{\rho}\left(x_{0}\right)}\left[\left(1+|D l|^{2}\right)^{\frac{m-2}{2}}|D u-D l|^{2}+|D u-D l|^{m}\right] d x d t \\
& \leq C_{1}\left[\left(1+|D l|^{2}\right)^{\frac{m-2}{2}} \int_{Q_{R}\left(z_{0}\right)} \frac{1}{|R-\rho|^{2}}|u-l|^{2} d z+\int_{Q_{R}\left(z_{0}\right)} \frac{1}{|R-\rho|^{m}}|u-l|^{m} d z\right] \\
& \quad+C_{2}\left[K(|l|)(1+|D l|)^{\frac{m}{2}}\right]^{\frac{2}{1-\beta}} \alpha_{n} R^{n+2+2 \beta}+C_{3}\left[a^{2}\left(1+\frac{1}{\mu}\right)^{2}|D l|^{2 m}+b^{2}\right] \alpha_{n} R^{n+4} .
\end{aligned}
$$

Then the desired result follows by taking the limit $\sigma \rightarrow 0$. 


\section{The proof of the main theorem}

The next lemma is a prerequisite for applying the $A$-caloric approximation technique.

Lemma 4.1 Let $u \in L^{m}\left(-T, 0 ; W^{1, m}\left(\Omega, R^{N}\right)\right) \cap L^{\infty}\left(Q_{T} ; R^{N}\right)$ be a weak solution to (1.9) under the assumptions (H1)-(H6). Then for any $M>0$, we have

$$
\begin{aligned}
& \left|f_{Q_{\rho}\left(z_{0}\right)}\left((u-l)^{i} \varphi_{t}^{i}-\frac{\partial A_{i}^{\alpha}}{\partial p_{\beta}^{j}}\left(z_{0}, l\left(z_{0}\right), D l\right)\left(D u^{i}-D l^{i}\right) D_{\alpha} \varphi^{i}\right) d z\right| \\
& \quad \leq C_{\mathrm{Eu}}\left(\omega(M+1, \Phi) \Phi^{\frac{1}{2}}+\Phi+\Psi+H(M) \rho^{\beta}\right) \sup _{Q_{\rho}\left(z_{0}\right)}|D \varphi|,
\end{aligned}
$$

for any $Q_{\rho}\left(z_{0}\right) \subset \subset Q_{T}$ and $\varphi \in C_{0}^{\infty}\left(Q_{\rho}\left(z_{0}\right), R^{N}\right)$ with $\rho \leq 1$ and any affine function $l(z)=$ $l(x)$ independent of time, satisfying $\left|l\left(z_{0}\right)\right|+|D l| \leq M$. Here $C_{\mathrm{Eu}}=C_{\mathrm{Eu}}(M, L, m)$ and we write

$$
\begin{aligned}
& \Phi=\Phi\left(z_{0}, \rho, D l\right)=\left(1+|D l|^{2}\right)^{\frac{m-2}{2}} f_{Q_{\rho}\left(z_{0}\right)}|D u-D l|^{2} d z+f_{Q_{\rho}\left(z_{0}\right)}|D u-D l|^{m} d z, \\
& \Psi\left(z_{0}, R, l\right)=\left(1+|D l|^{2}\right)^{\frac{m-2}{2}} f_{Q_{R}\left(z_{0}\right)} \frac{1}{|R-\rho|^{2}}|u-l|^{2} d z+f_{Q_{R}\left(z_{0}\right)} \frac{1}{|R-\rho|^{m}}|u-l|^{m} d z, \\
& \Psi=\Psi\left(z_{0}, \rho\right), \\
& H(s)=\left[\tilde{K}(s)(1+s)^{\frac{m}{2}}\right]^{\frac{2}{1-\beta}}, \quad \text { for } \tilde{K}(s)=\max \{K(s), a, b\} .
\end{aligned}
$$

Proof Without loss of generality, we can assume that $\sup _{Q_{\rho}\left(z_{0}\right)}|D \varphi| \leq 1$. From (1.12) and the fact that $f_{Q_{\rho}\left(z_{0}\right)} A_{i}^{\alpha}\left(z_{0}, l\left(z_{0}\right), D l\right) D_{\alpha} \varphi^{i} d z=0$ and $f_{Q_{\rho}\left(z_{0}\right)} l \varphi_{t} d z=0$, we deduce

$$
\begin{aligned}
& f_{Q_{\rho}\left(z_{0}\right)}\left((u-l)^{i} \varphi_{t}^{i}-\frac{\partial A_{i}^{\alpha}}{\partial p_{\beta}^{j}}\left(z_{0}, l\left(z_{0}\right), D l\right)\left(D u^{i}-D l^{i}\right) D_{\alpha} \varphi^{i}\right) d z \\
& =f_{Q_{\rho}\left(z_{0}\right)}\left[A_{i}^{\alpha}\left(z_{0}, l\left(z_{0}\right), D u\right)-\frac{\partial A_{i}^{\alpha}}{\partial p_{\beta}^{j}}\left(z_{0}, l\left(z_{0}\right), D l\right)\left(D u^{i}-D l^{i}\right)\right] D_{\alpha} \varphi^{i} d z \\
& \quad+f_{Q_{\rho}\left(z_{0}\right)}\left[A_{i}^{\alpha}(z, u, D u)-A_{i}^{\alpha}(z, l, D u)\right] D_{\alpha} \varphi^{i} d z \\
& \quad+f_{Q_{\rho}\left(z_{0}\right)}\left[A_{i}^{\alpha}(z, l, D u)-A_{i}^{\alpha}\left(z_{0}, l\left(z_{0}\right), D u\right)\right] D_{\alpha} \varphi^{i} d z \\
& \quad-f_{Q_{\rho}\left(z_{0}\right)} B_{i}(z, u, D u) \varphi^{i} d z \\
& =I+I I+I I I+I V .
\end{aligned}
$$

In turn, we split the first integral as follows:

$$
I=\frac{1}{\left|Q_{\rho}\left(z_{0}\right)\right|} \int_{s_{1}}(\cdots) d z+\frac{1}{\left|Q_{\rho}\left(z_{0}\right)\right|} \int_{s_{2}}(\cdots) d z=I_{1}+I_{2}
$$

and $s_{1}=Q_{\rho}\left(z_{0}\right) \cap\{z:|D u-D l| \leq 1\}, s_{2}=Q_{\rho}\left(z_{0}\right) \cap\{z:|D u-D l|>1\}$. 
We proceed estimating the two resulting pieces. As for $I_{1}$, using (H6), the fact that $s \mapsto$ $\omega^{m}(t, s)$ is concave and Jensen's inequality (note that $\frac{m-1}{m}>\frac{1}{2}$ ), we get

$$
\begin{aligned}
I_{1}= & \frac{1}{\left|Q_{\rho}\left(z_{0}\right)\right|} \int_{s_{1}} \int_{0}^{1}\left[\frac{\partial A_{i}^{\alpha}}{\partial p_{\beta}^{j}}\left(z_{0}, l\left(z_{0}\right), D l+\tau(D u-D l)\right)\right. \\
& \left.-\frac{\partial A_{i}^{\alpha}}{\partial p_{\beta}^{j}}\left(z_{0}, l\left(z_{0}\right), D l\right)\right] d \tau(D u-D l) D_{\alpha} \varphi^{i} d z \\
\leq & L f_{Q_{\rho}\left(z_{0}\right)}\left(1+|D l|^{2}+|D u-D l|^{2}\right)^{\frac{m-2}{2}} \omega\left(M+1,|D u-D l|^{2}\right)|D u-D l| d z \\
\leq & L\left(1+|D l|^{2}\right)^{\frac{m-2}{2}}\left(f_{Q_{\rho}\left(z_{0}\right)} \omega^{m}\left(M+1,|D u-D l|^{2}\right) d z\right)^{\frac{1}{m}} \\
& \cdot\left[f_{Q_{\rho}\left(z_{0}\right)}\left(\left(1+|D u-D l|^{2}\right)^{\frac{m-2}{2}}|D u-D l|\right)^{\frac{m-1}{m-1}} d z\right]^{\frac{m-1}{m}} \\
\leq & L\left(1+|D l|^{2}\right)^{\frac{m-2}{2}} \omega\left(M+1, f_{Q_{\rho}\left(z_{0}\right)}^{\left.|D u-D l|^{2} d z\right)}\right. \\
& \cdot\left\{\left[\left(f_{Q_{\rho}\left(z_{0}\right)}|D u-D l|^{2} d z\right)^{\frac{m}{2(m-1)}}\right]^{\frac{m-1}{m}}\right. \\
& \left.+\left(f_{Q_{\rho}\left(z_{0}\right)}|D u-D l|^{m} d z\right)^{\frac{m-1}{m}}\right\} \\
\leq & L\left(1+|D l|^{2}\right)^{\frac{m-2}{2}} \omega\left(M+1, f_{Q_{\rho}\left(z_{0}\right)}^{\left.|D u-D l|^{2} d z\right)}\right. \\
& \cdot\left[f_{Q_{\rho}\left(z_{0}\right)}|D u-D l|^{2} d z+f_{Q_{\rho}\left(z_{0}\right)}^{\left.|D u-D l|^{m} d z\right]^{\frac{1}{2}} .}\right.
\end{aligned}
$$

To estimate $I_{2}$, we preliminarily observe that, using Hölder inequality,

$$
\begin{aligned}
\left|s_{2}\right| & \leq \int_{s_{2}}|D u-D l| d z \leq\left(\int_{s_{2}} d z\right)^{\frac{1}{2}}\left(\int_{s_{2}}|D u-D l|^{2} d z\right)^{\frac{1}{2}} \\
& \leq \sqrt{\left|s_{2}\right|}\left(\int_{Q_{\rho}}|D u-D l|^{2} d z\right)^{\frac{1}{2}}
\end{aligned}
$$

and therefore

$$
\frac{\sqrt{\left|s_{2}\right|}}{\sqrt{\left|Q_{\rho}\left(z_{0}\right)\right|}} \leq\left(f_{Q_{\rho}}|D u-D l|^{2} d z\right)^{\frac{1}{2}} .
$$

Similarly, we also have

$$
\frac{\sqrt{\left|s_{2}\right|}}{\sqrt{\left|Q_{\rho}\left(z_{0}\right)\right|}} \leq\left(f_{Q_{\rho}}|D u-D l|^{m} d z\right)^{\frac{1}{2}} .
$$


Using (H1), (H2) and the previous inequality, we then conclude the estimate of $I_{2}$ as follows:

$$
\begin{aligned}
\left|I_{2}\right| \leq & \frac{L}{\left|Q_{\rho}\left(z_{0}\right)\right|} \int_{s_{2}}\left|(1+|D u|)^{\frac{m}{2}}+\left(1+|D l|^{2}\right)^{\frac{m-2}{2}}\right| D u-D l|| d z \\
\leq & \frac{L}{\left|Q_{\rho}\left(z_{0}\right)\right|} \int_{s_{2}}\left|(1+|D l|)^{\frac{m}{2}}(1+|D u-D l|)^{\frac{m}{2}}\right| d z \\
& +\frac{L}{\left|Q_{\rho}\left(z_{0}\right)\right|}\left(\int_{s_{2}}\left(1+|D l|^{2}\right)^{\frac{m-2}{2}} d z\right)^{\frac{1}{2}}\left(\int_{s_{2}}\left(1+|D l|^{2}\right)^{\frac{m-2}{2}}|D u-D l|^{2} d z\right)^{\frac{1}{2}} \\
\leq & L(M+1)^{\frac{m}{2}} \frac{\sqrt{\left|s_{2}\right|}}{\sqrt{\left|Q_{\rho}\left(z_{0}\right)\right|}}\left(f_{Q_{\rho}\left(z_{0}\right)}|D u-D l|^{m} d z\right)^{\frac{1}{2}}+\frac{L\left|s_{2}\right|}{\left|Q_{\rho}\left(z_{0}\right)\right|}(1+M)^{\frac{m}{2}} \\
& +\frac{L \sqrt{\left|s_{2}\right|}}{\sqrt{\left|Q_{\rho}\left(z_{0}\right)\right|}}\left(1+|D l|^{2}\right)^{\frac{m-2}{4}}\left(f_{Q_{\rho}\left(z_{0}\right)}\left(1+|D l|^{2}\right)^{\frac{m-2}{2}}|D u-D l|^{2} d z\right)^{\frac{1}{2}} \\
\leq & 2 L(1+M)^{\frac{m}{2}} \\
& \times\left[\left(1+|D l|^{2}\right)^{\frac{m-2}{2}} f_{Q_{\rho}\left(z_{0}\right)}|D u-D l|^{2} d z+f_{Q_{\rho}\left(z_{0}\right)}|D u-D l|^{m} d z\right] .
\end{aligned}
$$

Combining the estimates found for $I_{1}$ and $I_{2}$, we have

$$
|I| \leq L\left(1+M^{2}\right)^{\frac{m-2}{2}} \omega(M+1, \Phi) \sqrt{\Phi}+2 L(1+M)^{\frac{m}{2}} \Phi .
$$

For the remaining pieces, using $\left(\mathrm{H} 4^{\prime}\right)$, we deduce

$$
\begin{aligned}
|I I| \leq & K\left(\left|l\left(z_{0}\right)\right|\right) f_{Q_{\rho}\left(z_{0}\right)}|u-l|^{\beta}(1+|D l|+|D u-D l|)^{\frac{m}{2}} d z \\
\leq & 2^{\frac{m}{2}}\left[f_{Q_{\rho}\left(z_{0}\right)} \frac{1}{\rho^{2}}|u-l|^{2} d z+f_{Q_{\rho}\left(z_{0}\right)}|D u-D l|^{m} d z\right. \\
& \left.+\left[K\left(\left|l_{0}\right|\right)(1+|D l|)^{\frac{m}{2}}\right]^{\frac{2}{1-\beta}} \rho^{\frac{2 \beta}{2-\beta}}\right] .
\end{aligned}
$$

Here we have used that $K \geq 1$ and the assumption that $\rho \leq 1$. Using again $\left(\mathrm{H} 4^{\prime}\right)$ and Young's inequality, we estimate

$$
\begin{aligned}
|I I I| & \leq K(|l|) f_{Q_{\rho}\left(z_{0}\right)} \rho^{\beta}(1+|D l|)^{\beta}(1+|D l|+|D u-D l|)^{\frac{m}{2}} d z \\
& \leq 2^{\frac{m}{2}}\left(H(M) R^{\beta}+f_{Q_{\rho}\left(z_{0}\right)}|D u-D l|^{m} d z\right)
\end{aligned}
$$

and

$$
|I V| \leq f_{Q_{\rho}\left(z_{0}\right)} b R d z+2^{m-1} f_{Q_{\rho}\left(z_{0}\right)} a R|D l|^{m} d z+2^{m-1} a f_{Q_{\rho}\left(z_{0}\right)} R|D u-D l|^{m} d z
$$


Noting the definition of $H$ and combining the estimates just found for $I, I I, I I I$ and $I V$, we obtain

$$
\begin{aligned}
& \left|f_{Q_{\rho}\left(z_{0}\right)}\left((u-l)^{i} \varphi_{t}^{i}-\frac{\partial A_{i}^{\alpha}}{\partial p_{\beta}^{j}}\left(z_{0}, l\left(z_{0}\right), D l\right)\left(D u^{i}-D l^{i}\right) D_{\alpha} \varphi^{i}\right) d z\right| \\
& \quad \leq C(L, M, m)\left[\omega(M+1, \Phi) \Phi^{\frac{1}{2}}+\Phi+\Psi+H(M) \rho^{\beta}\right] .
\end{aligned}
$$

A simple scaling argument yields the result for general $\varphi$.

The next lemma is a standard estimate for weak solutions to linear parabolic systems with constant coefficients [15], Lemma 5.1.

Lemma 4.2 Leth $\in L^{2}\left(t_{0}-\rho^{2}, t_{0} ; W^{1,2}\left(B_{\rho}\left(x_{0}\right), R^{N}\right)\right)$ be a weak solution in $Q_{\rho}\left(z_{0}\right)=B_{\rho}\left(x_{0}\right) \times$ $\left(t_{0}-\rho^{2}, t_{0}\right)$ of the following linear parabolic system with constant coefficients:

$$
f_{Q_{\rho}\left(z_{0}\right)}\left(h^{i} \varphi_{t}^{i}-A_{i}^{\alpha}\left(D h, D_{\alpha} \varphi^{i}\right)\right) d z=0, \quad \forall \varphi \in C_{0}^{\infty}\left(Q_{\rho}\left(z_{0}\right), R^{N}\right),
$$

where the coefficients $A_{i}^{\alpha}$ satisfy $A_{i}^{\alpha}(p, p) \geq \lambda|p|^{2}, A_{i}^{\alpha}(p, \tilde{p}) \leq L|p||\tilde{p}|$ for any $p, \tilde{p} \in R^{n N}$. Then $h$ is smooth in $Q_{\rho}\left(z_{0}\right)$ and there exists a constant $C_{\mathrm{pa}}=C_{\mathrm{pa}}(n, N, L / \lambda) \geq 1$ such that

$$
\widetilde{\psi}\left(z_{0}, \theta \rho\right) \leq C_{\mathrm{pa}} \theta^{2} \widetilde{\psi}\left(z_{0}, \rho\right), \quad \forall 0<\theta<1
$$

Here we write

$$
\widetilde{\psi}\left(z_{0}, \sigma\right)=\frac{1}{\sigma^{2}} f_{Q_{\rho}\left(z_{0}\right)}\left|h-(h)_{z_{0}, \sigma}-(D h)_{z_{0}, \sigma}\left(x-x_{0}\right)\right|^{2} d z .
$$

In the following we consider a weak solution $u$ of the nonlinear parabolic system (1.9) on a fixed sub-cylinder $Q_{\rho}\left(z_{0}\right) \subset Q_{T}$ and $\rho \leq 1$.

Lemma 4.3 Given $M>0$ and $0<\beta<\alpha<1$, there exist $\theta \in\left(0, \frac{1}{2}\right)$ and $\delta \in(0,1]$ depending only on $n, N, \lambda, L, \beta, \alpha$ and $m$ such that if

$$
\omega\left(M+1, \tilde{\Psi}\left(z_{0}, \rho, l_{z_{0}, \rho}\right)\right)+\sqrt{\tilde{\Psi}\left(z_{0}, \rho, l_{z_{0}, \rho}\right)} \leq \frac{\delta}{2}
$$

on $Q_{\rho}\left(z_{0}\right) \subset Q_{T}$ for some $0<\rho \leq 1$ and such if

$$
\left|l_{z_{0}, \rho}\left(z_{0}\right)\right|+\left|D l_{z_{0}, \rho}\right| \leq M
$$

then

$$
\tilde{\Psi}\left(z_{0}, \theta \rho, l_{z_{0}, \theta \rho}\right) \leq \theta^{2 \alpha} \tilde{\Psi}\left(z_{0}, \rho, l_{z_{0}, \rho}\right)+C_{6} \rho^{2 \beta} H^{2}(M)
$$

for

$$
\tilde{\Psi}\left(z_{0}, \rho, l_{z_{0}, \rho}\right)=\Psi\left(z_{0}, \rho, l_{z_{0}, \rho}\right)+H^{2}(M) \rho^{2 \beta} .
$$


Proof Given $M>1$. And we shall always consider $\rho \leq 1$. We first want to apply Lemma 4.1 on $Q_{\rho / 2}\left(z_{0}\right)$ to $u-l$, where $l(z)=l(x)$ is an affine function independent of $t$ satisfying $\left|l\left(z_{0}\right)\right|+$ $|D l| \leq M$. We observe that $\Psi$ has the following property:

$$
\begin{aligned}
\Psi\left(z_{0}, \rho / 2, l\right) & \leq 2^{n+m+2}\left[\left(1+|D l|^{2}\right)^{\frac{m-2}{2}} f_{Q_{\rho}\left(z_{0}\right)}\left|\frac{u-l}{\rho}\right|^{2} d z+f_{Q_{\rho}\left(z_{0}\right)}\left|\frac{u-l}{\rho}\right|^{m} d z\right] \\
& =2^{n+m+2} \Psi\left(z_{0}, \rho, l\right) .
\end{aligned}
$$

From Caccioppoli's second inequality, we infer

$$
\Phi\left(z_{0}, \rho / 2, l\right) \leq C_{\mathrm{cac}}\left[2^{m} \Psi\left(z_{0}, \rho, l\right)+2 H(M) \rho^{2 \beta}\right]=\tilde{C}_{\mathrm{cac}} \tilde{\Psi}\left(z_{0}, \rho, l\right) .
$$

From Lemma 4.1 we therefore get, for any $\varphi \in C_{0}^{\infty}\left(Q_{\rho / 2}\left(z_{0}\right), R^{N}\right)$, that

$$
\begin{aligned}
& \left|f_{Q_{\rho / 2}\left(x_{0}\right)}\left[(u-l)^{i} \varphi_{t}^{i}-\frac{\partial A_{i}^{\alpha}}{\partial p_{\beta}^{j}}\left(z_{0}, l\left(z_{0}\right), D l\right) D(u-l)^{i} D_{\alpha} \varphi^{i}\right] d z\right| \\
& \quad \leq \tilde{C}_{\mathrm{Eu}}\left[\omega(M+1, \tilde{\Psi}) \sqrt{\tilde{\Psi}}+\tilde{\Psi}+\rho^{\beta} H(M)\right] \cdot \sup _{Q_{\rho / 2}\left(z_{0}\right)}|D \varphi|,
\end{aligned}
$$

where $\tilde{C}_{\mathrm{Eu}}=\tilde{C}_{\mathrm{Eu}}(L, M, m)$.

For given $\varepsilon>0$ to be specified later, we let $\delta=\delta(n, N, \lambda, L, \varepsilon) \in(0,1]$ to be constant from Lemma 2.3. Define $\gamma=\tilde{C}_{\mathrm{Eu}} \sqrt{\Psi\left(z_{0}, \rho\right)+4 \delta^{-2} H^{2}(M) \rho^{2 \beta}}$ and $w=\gamma^{-1}(u-l)$.

Then from (4.3) we deduce that, for all $\varphi \in C_{0}^{\infty}\left(Q_{\rho / 2}\left(z_{0}\right), R^{N}\right)$, the following holds:

$$
\begin{aligned}
& f_{Q_{\rho / 2}\left(x_{0}\right)}\left[w^{i} \varphi_{t}^{i}-\frac{\partial A_{i}^{\alpha}}{\partial p_{\beta}^{j}}\left(z_{0}, l\left(z_{0}\right), D l\right) D w D_{\alpha} \varphi^{i}\right] d z \\
& \quad \leq\left[\omega\left(M+1, \tilde{\Psi}\left(z_{0}, \rho, l_{z_{0}, \rho}\right)\right)+\sqrt{\tilde{\Psi}\left(z_{0}, \rho, l_{z_{0}, \rho}\right)}+\frac{\delta}{2}\right] \sup _{Q_{\rho / 2}\left(z_{0}\right)}|D \varphi| .
\end{aligned}
$$

Moreover, we estimate, using Caccioppoli's second inequality, (4.1) and (4.2),

$$
(\rho / 2)^{-2} f_{Q_{\rho / 2}\left(x_{0}\right)}|w|^{2} d z+f_{Q_{\rho / 2}\left(x_{0}\right)}|D w|^{2} d z \leq \frac{2^{n+m+2}+\tilde{C}_{\mathrm{cac}}}{\tilde{C}_{\mathrm{Eu}}^{2}} \leq 1,
$$

provided we have chosen $\tilde{C}_{\mathrm{Eu}} \gg 1$ large enough.

Assuming the smallness condition,

$$
\omega\left(M+1, \tilde{\Psi}\left(z_{0}, \rho, l_{z_{0}, \rho}\right)\right)+\sqrt{\tilde{\Psi}\left(z_{0}, \rho, l_{z_{0}, \rho}\right)} \leq \frac{\delta}{2},
$$

satisfied. Then (4.4) and (4.5) allow us to apply Lemma 2.4, i.e., they yield the existence of $h \in L^{2}\left(t_{0}-\rho^{2}, t_{0} ; W^{1,2}\left(B_{\rho}\left(x_{0}\right), R^{N}\right)\right)$ solving the $\frac{\partial A_{j}^{\alpha}}{\partial p_{\beta}^{j}}$-heat equation on $Q_{\rho / 2}\left(z_{0}\right)$ and satisfying

$$
(\rho / 2)^{-2} f_{Q_{\rho / 2}\left(x_{0}\right)}|h|^{2} d z+f_{Q_{\rho / 2}\left(x_{0}\right)}|D h|^{2} d z \leq 1
$$


and

$$
(\rho / 2)^{-2} f_{Q_{\rho} / 2\left(x_{0}\right)}|w-h|^{2} d z \leq \varepsilon .
$$

From Lemma 4.2 we recall that $h$ satisfies, for any $0<\theta<1$, the a priori estimate (note that $\left.C_{\mathrm{pa}}=C_{\mathrm{pa}}(n, N, \lambda, L) \geq 1\right)$

$$
\begin{aligned}
& (\theta \rho / 2)^{-2} f_{Q_{\theta \rho / 2}\left(x_{0}\right)}\left|h-(h)_{z_{0}, \theta \rho / 2}-(D h)_{z_{0}, \theta \rho / 2}\left(x-x_{0}\right)\right|^{2} d z \\
& \leq C_{\mathrm{pa}} \theta^{2}(\rho / 2)^{-2} f_{Q_{\rho / 2}\left(x_{0}\right)}\left|h-(h)_{z_{0}, \rho / 2}-(D h)_{z_{0}, \rho / 2}\left(x-x_{0}\right)\right|^{2} d z \\
& \leq 3 C_{\mathrm{pa}} \theta^{2}\left[(\rho / 2)^{-2}\left(f_{Q_{\rho / 2}\left(x_{0}\right)}|h|^{2}+\left|(h)_{z_{0}, \rho / 2}\right|^{2}\right)+\left|(D h)_{z_{0}, \rho / 2}\right|^{2}\right] \\
& \leq 6 C_{\mathrm{pa}} \theta^{2}\left[(\rho / 2)^{-2} f_{Q_{\rho / 2}\left(x_{0}\right)}|h|^{2} d z+f_{Q_{\rho / 2}\left(x_{0}\right)}|D h|^{2} d z\right] \\
& \leq 6 C_{\mathrm{pa}} \theta^{2} .
\end{aligned}
$$

Here we have used that $\left|(h)_{z_{0}, \rho / 2}\right|^{2} \leq f_{Q_{\rho / 2}\left(x_{0}\right)}|h|^{2} d z$, and $\left|(D h)_{z_{0}, \rho / 2}\right|^{2} \leq f_{Q_{\rho / 2}\left(x_{0}\right)}|D h|^{2} d z$ and (4.7). Combining the previous estimate with (4.8), we deduce

$$
\begin{aligned}
& (\theta \rho / 2)^{-2} f_{Q_{\theta \rho / 2}\left(x_{0}\right)}\left|w-(h)_{z_{0}, \theta \rho / 2}-(D h)_{z_{0}, \theta \rho / 2}\left(x-x_{0}\right)\right|^{2} d z \\
& \leq 2(\theta \rho / 2)^{-2} \\
& \quad \times\left[f_{Q_{\theta \rho / 2}\left(x_{0}\right)}|w-h|^{2} d z+f_{Q_{\theta \rho / 2}\left(x_{0}\right)}\left|h-(h)_{z_{0}, \theta \rho / 2}-(D h)_{z_{0}, \theta \rho / 2}\left(x-x_{0}\right)\right|^{2} d z\right] \\
& \leq 12 C_{\mathrm{pa}}\left(\theta^{-n-4} \varepsilon+\theta^{2}\right) .
\end{aligned}
$$

Recalling back $(u-l)$ via $w=\frac{u-l}{\gamma}$, we arrive at

$$
\begin{aligned}
& (\theta \rho)^{-2} f_{Q_{\theta \rho / 2}\left(x_{0}\right)}\left|u-l-\gamma\left((h)_{z_{0}, \theta \rho / 2}+(D h)_{z_{0}, \theta \rho / 2}\left(x-x_{0}\right)\right)\right|^{2} d z \\
& \leq 12 C_{\mathrm{pa}}\left(\theta^{-n-4} \varepsilon+\theta^{2}\right) \gamma^{2} .
\end{aligned}
$$

Next we use the minimizing property of $l_{z_{0}, \theta \rho / 2}$

$$
(\theta \rho / 2)^{-2} f_{Q_{\theta \rho / 2}\left(z_{0}\right)}\left|u-l_{z_{0}, \theta \rho / 2}\right|^{2} d z \leq 12 C_{\mathrm{pa}}\left(\theta^{-n-4} \varepsilon+\theta^{2}\right) \gamma^{2}
$$

At the same time, from (4.11), we can see that: For $2 \leq m \leq n+2(n \geq 3)$, we have $2<$ $m<m^{*}$, where

$$
m^{*}= \begin{cases}\frac{m(n+2)}{n-m+2} & \text { if } n+2>m \\ m^{*}>m & \text { if } m=n+2\end{cases}
$$

with $\frac{1}{m^{*}}<\frac{1}{m}<\frac{1}{2}$. Therefore we can find $s \in[0,1]$ such that $\frac{1}{m}=\frac{1-s}{2}+\frac{s}{m^{*}}$. 
Using Sobolev's, Caccioppoli's and Young's inequalities together with (4.11), we have

$$
\begin{aligned}
(\theta \rho / 2)^{-m} & f_{Q_{\theta \rho / 2}\left(z_{0}\right)}\left|u-l-\gamma\left((h)_{z_{0}, \theta \rho / 2}+(D h)_{z_{0}, \theta \rho / 2}\left(x-x_{0}\right)\right)\right|^{m} d z \\
\leq & (\theta \rho / 2)^{-m s}\left[(\theta \rho / 2)^{-2} f_{Q_{\theta \rho / 2}\left(z_{0}\right)}\left|u-l-\gamma\left((h)_{z_{0}, \theta \rho / 2}+(D h)_{z_{0}, \theta \rho / 2}\left(x-x_{0}\right)\right)\right|^{2} d z\right]^{\frac{(1-s) m}{2}} \\
& \cdot\left[f_{Q_{\theta \rho / 2}\left(z_{0}\right)}\left|u-l-\gamma\left((h)_{z_{0}, \theta \rho / 2}+(D h)_{z_{0}, \theta \rho / 2}\left(x-x_{0}\right)\right)\right|^{m^{*}} d z\right]^{\frac{m s}{m^{*}}} \\
\leq & {\left[12 C_{\mathrm{pa}}\left(\theta^{-n-4} \varepsilon+\theta^{2}\right) \gamma^{2}\right]^{\frac{(1-s) m}{2}}\left[f_{Q_{\theta \rho / 2}\left(z_{0}\right)}\left|D u-D l-\gamma(D h)_{z_{0}, \theta \rho / 2}\right|^{m} d z\right]^{s} }
\end{aligned}
$$

Using Lemma 2.5, Caccioppoli's inequality, (4.4), (4.6), (4.12) and Young's inequality, we obtain

$$
\begin{aligned}
& \left(\int_{Q_{\theta \rho / 2}\left(z_{0}\right)}\left|D u-D l-\gamma(D h)_{z_{0}, \theta \rho / 2}\right|^{m} d z\right)^{\frac{1}{m}} \\
& =\left|Q_{\theta \rho / 2}\right|^{\frac{1}{m}}\left[\left(f_{Q_{\theta \rho / 2}\left(z_{0}\right)}\left|D u-D l-(D h)_{z_{0}, \rho}\right|^{m} d z\right)^{\frac{1}{m}}\right. \\
& \left.\quad+\left(f_{Q_{\theta \rho / 2}\left(z_{0}\right)}\left|(D h)_{z_{0}, \rho}-\gamma(D h)_{z_{0}, \theta \rho / 2}\right|^{m} d z\right)^{\frac{1}{m}}\right] \\
& \leq\left|Q_{\theta \rho / 2}\right|^{\frac{1}{m}}\left[\left(C_{\mathrm{cac}}\left(\Psi+H(M)(\theta \rho)^{2 \beta}\right)\right)^{\frac{1}{m}}\right. \\
& \left.\quad+\left(\frac{4 n(n+2)}{(\theta \rho)^{2}} f_{Q_{\theta \rho / 2}\left(z_{0}\right)}\left|u-l-\gamma(D u)_{z_{0}, \rho}\left(x-x_{0}\right)\right|^{2} d z\right)^{\frac{1}{2}}\right] \\
& \leq\left|Q_{\theta \rho / 2}\right|^{\frac{1}{m}}\left[\left(C_{\mathrm{cac}}\left(\Psi+H(M)(\theta \rho)^{2 \beta}\right)\right)^{\frac{1}{m}}+\frac{2 \sqrt{n(n+2)}}{\theta \rho} \Psi\right] \\
& \leq\left|Q_{\theta \rho / 2}\right|^{\frac{1}{m}} \theta^{-1}\left[\tilde{C}_{\mathrm{cac}}^{\frac{1}{m}}+2 \sqrt{n(n+2)}\right] \gamma^{\frac{2}{m}} .
\end{aligned}
$$

From (4.12) and (4.13), we conclude

$$
\begin{aligned}
& (\theta \rho / 2)^{-m} f_{Q_{\theta \rho / 2}\left(z_{0}\right)}\left|u-l-\gamma\left((h)_{z_{0}, \theta \rho / 2}+(D h)_{z_{0}, \theta \rho / 2}\left(x-x_{0}\right)\right)\right|^{m} d z \\
& \quad \leq C_{4}\left[\frac{(1-s) m}{2}\left(\theta^{-n-4} \varepsilon+\theta^{2}\right) \gamma^{2}+\frac{s m}{m^{*}} \theta^{-\frac{(n+m) m^{*}}{m}} \gamma^{\frac{2 m^{*}}{m}}\right] \\
& \leq C_{4} \theta^{2} \gamma^{2}
\end{aligned}
$$

provided $\gamma^{2\left(m^{*}-m\right) / m} \leq \theta^{2+(n+m) m^{*} / m}$ and we fixed $\varepsilon=\theta^{n+6}$. That it is to say,

$$
(\theta \rho / 2)^{-m} f_{Q_{\theta \rho / 2}\left(z_{0}\right)}\left|u-l_{z_{0}, \theta \rho / 2}\right|^{m} d z \leq C_{4} \theta^{2} \gamma^{2} .
$$


Combining (4.11) and (4.15) yields the desired estimate

$$
\Psi\left(z_{0}, \theta \rho / 2, l_{z_{0}, \theta \rho / 2}\right) \leq C_{5} \theta^{2}\left(\Psi\left(z_{0}, \rho, l\right)+4 \delta^{-2} H^{2}(M) \rho^{2 \beta}\right)
$$

for $C_{5}=C_{4}+12 C_{\mathrm{pa}}$. Given $\beta<\alpha<1$, we choose $0<\theta<1$ such that $2^{2 \alpha} C_{5} \theta^{2} \leq \theta^{2 \alpha}$ with $\theta=\theta(n, m, N, \lambda, L, \alpha, \beta)$. This also fixes the constants $\varepsilon=\varepsilon(n, m, N, \lambda, L, \alpha, \beta)$ and $\delta=\delta(n, m, N, \lambda, L, \alpha, \beta) \in(0,1]$. Thus we have shown Lemma 4.3.

In the following, we want to iterate Lemma 4.3. That is,

Lemma 4.4 For $M>1$ and $Q_{\rho}\left(z_{0}\right) \subset \subset Q_{T}$, suppose that the conditions

(i) $\left|l_{z_{0}, \rho}\right|+\left|(D l)_{z_{0}, \rho}\right| \leq M$

(ii) $\rho \leq \rho_{0}(M)$;

(iii) $\tilde{\Psi}(\rho) \leq \tilde{\Psi}_{0}(M)$

are satisfied. Then, for every $j \in N$, we have

$$
\tilde{\Psi}\left(z_{0}, \theta^{j} \rho, l_{z_{0}, \theta \rho}\right) \leq \theta^{2 \alpha j} \tilde{\Psi}\left(z_{0}, \rho, l_{z_{0}, \rho}\right)+C_{6}(M)\left(\theta^{j} \rho\right)^{2 \beta} H^{2}(M)
$$

and

$$
\left|l_{z_{0}, \theta^{j} \rho}\right|+\left|(D l)_{z_{0}, \theta^{j} \rho}\right| \leq 2 M \text {. }
$$

Moreover, the limit

$$
\Gamma_{z_{0}}=\lim _{j \rightarrow \infty}(D u)_{z_{0}, \theta^{j} \rho / 2}
$$

exists, and the estimate

$$
\begin{aligned}
& \left(1+|D l|^{2}\right)^{\frac{m-2}{2}} f_{Q_{r}\left(z_{0}\right)}\left|D u-\Gamma_{z_{0}}\right|^{2} d z+f_{Q_{r}\left(z_{0}\right)}\left|D u-\Gamma_{z_{0}}\right|^{m} d z \\
& \leq C\left[\left(\frac{r}{\rho / 2}\right)^{2 \alpha} \Psi\left(z_{0}, \rho, l_{z_{0}, \rho}\right)+r^{2 \beta} H^{2}(M)\right]
\end{aligned}
$$

is valid for a constant $C=C(n, N, \lambda, \alpha, L, \beta, M, m)$.

Proof For fixed $z_{0}$ we shall denote $l_{z_{0}, \rho} \equiv l_{\rho}$. For given $M>1$ (and $\beta<\alpha<1$ ), we determine $\delta=\delta(2 M), \theta=\theta(2 M)$ and $C_{6}=C_{6}(2 M)$ according to Lemma 4.3. Then we can find $\tilde{\Psi}_{0}(M)>0$ sufficiently small such that

$$
\omega\left(M+1,2 \tilde{\Psi}_{0}(M)\right)+\sqrt{\tilde{\Psi}_{0}(M)} \leq \frac{\delta}{2}
$$

and

$$
\tilde{\Psi}_{0}(M) \leq \frac{M^{2} \theta^{n+4}\left(1-\theta^{\alpha}\right)^{2}}{4(n+2)^{2}} .
$$


Given this, we can also find $\rho_{0}(M) \in(0,1]$ so small that, writing

$$
C_{7}(M)=\frac{C_{6}(2 M)}{\theta^{2 \beta}-\theta^{2 \alpha}}
$$

we have

$$
C_{7}(M) \rho_{0}(M)^{2 \beta} H^{2}(M) \leq \min \left\{\frac{\delta^{2}}{16}, \tilde{\Psi}_{0}(M), \frac{M^{2} \theta^{n+4}\left(1-\theta^{\beta}\right)^{2}}{4(n+2)^{2}}\right\} .
$$

Now, suppose that the conditions (i), (ii) and (iii) are satisfied on $Q_{\rho}\left(z_{0}\right) \subset Q_{T}$. Then, for $j=1,2,3, \ldots$, we shall show

(I) $j \quad \tilde{\Psi}\left(z_{0}, \theta^{j} \rho, l_{z_{0}, \theta \rho}\right) \leq \theta^{2 \alpha j} \tilde{\Psi}\left(z_{0}, \rho, l_{z_{0}, \rho}\right)+C_{7}(M)\left(\theta^{j} \rho\right)^{2 \beta} H^{2}(M)$,

(II) $)_{j} \quad\left|l_{z_{0}, \theta^{j} \rho}\left(z_{0}\right)\right|+\left|(D l)_{z_{0}, \theta^{j} \rho}\right| \leq 2 M$.

Note first that $(\mathrm{I})_{j}$ combined with (ii), (iii) and (4.19) yields

$$
(\mathrm{I})^{j} \quad \tilde{\Psi}\left(\theta^{j} \rho\right) \leq 2 \tilde{\Psi}_{0}(M) .
$$

Moreover, we have $\rho \leq \rho_{0}(M) \leq 1$ and $\left|l_{z_{0}, \rho}\right|+\left|(D l)_{z_{0}, \rho}\right| \leq M$. There we can apply Lemma 4.3 to conclude that $(\mathrm{I})_{1}$ holds. Furthermore, using Lemma 2.5, (iii) and (4.18), we deduce

$$
\begin{aligned}
\left|l_{z_{0}, \theta \rho}\right|+\left|(D l)_{z_{0}, \theta \rho}\right| & \leq M+\left|f_{Q_{\theta \rho}\left(z_{0}\right)}\left(u-u_{\rho}\right) d z\right|+\left[\frac{n(n+2)}{(\theta \rho)^{2}} f_{Q_{\theta \rho}\left(z_{0}\right)}\left|u-l_{\rho}\right|^{2} d z\right]^{\frac{1}{2}} \\
& \leq M+\left[f_{Q_{\theta \rho}\left(z_{0}\right)}\left|u-l_{\rho}\right|^{2} d z\right]^{\frac{1}{2}}+\left[\frac{n(n+2)}{(\theta \rho)^{2}} f_{Q_{\theta \rho}\left(z_{0}\right)}\left|u-l_{\rho}\right|^{2} d z\right]^{\frac{1}{2}} \\
& \leq M+\frac{1+\sqrt{n(n+2)}}{\sqrt{\theta^{n+4}}} \tilde{\Psi}^{\frac{1}{2}}\left(z_{0}, \rho\right) \\
& \leq 2 M,
\end{aligned}
$$

i.e., (II) $)_{1}$ holds. We now assume that $(\mathrm{I})_{\iota}$ and $(\mathrm{II})_{\iota}$ for $\iota=1,2, \ldots, j-1$ hold. We can apply Lemma 4.3 to calculate

$$
\begin{aligned}
\tilde{\Psi}\left(\theta^{j} \rho\right) & \leq \theta^{2 \alpha j} \tilde{\Psi}(\rho)+C_{6}(2 M)\left(\theta^{j} \rho\right)^{2 \beta} \theta^{-2 \beta} \sum_{\iota=0}^{j-1} \theta^{2(\alpha-\beta) \iota} \\
& \leq \theta^{2 \alpha j} \tilde{\Psi}(\rho)+\frac{C_{6}(2 M)}{\theta^{2 \beta}-\theta^{2 \alpha}}\left(\theta^{j} \rho\right)^{2 \beta} \\
& =\theta^{2 \alpha j} \tilde{\Psi}(\rho)+C_{7}(2 M)\left(\theta^{j} \rho\right)^{2 \beta},
\end{aligned}
$$

showing $(\mathrm{I})_{j}$. To show $(\mathrm{II})_{j}$ we estimate

$$
\begin{aligned}
& \left|l_{z_{0}, \theta^{j} \rho}\right|+\left|(D l)_{z_{0}, \theta^{j} \rho}\right| \\
& \quad \leq M+\sum_{\imath=1}^{j}\left[f_{Q_{\theta^{\imath} \rho}\left(z_{0}\right)}\left|u-l_{\theta^{l-1} \rho}\right|^{2} d z\right]^{\frac{1}{2}}+\sum_{\imath=1}^{j}\left[\frac{n(n+2)}{\left(\theta^{\imath} \rho\right)^{2}} f_{Q_{\theta^{\imath} \rho}\left(z_{0}\right)}\left|u-l_{\theta^{l-1} \rho}\right|^{2} d z\right]^{\frac{1}{2}}
\end{aligned}
$$




$$
\begin{aligned}
& \leq M+\frac{1+\sqrt{n(n+2)}}{\sqrt{\theta^{n+4}}} \sum_{\imath=1}^{j} \Psi^{\frac{1}{2}}\left(z_{0}, \theta^{\iota-1} \rho\right) \\
& \leq M+\frac{\sqrt{n(n+2)}}{\sqrt{\theta^{n+4}}} \sum_{\imath=0}^{j-1} \sqrt{\theta^{2 \alpha \iota} \tilde{\Psi}(\rho)+C_{7}(2 M)\left(\theta^{\iota} \rho\right)^{2 \beta}} \\
& \leq M+\frac{n+2}{\sqrt{\theta^{n+4}}}\left(\frac{\sqrt{\tilde{\Psi}(\rho)}}{1-\theta^{\alpha}}+\frac{\sqrt{C_{7}(M) \rho^{2 \beta}}}{1-\theta^{\beta}}\right) \\
& \leq 2 M .
\end{aligned}
$$

Here we have used in turn Lemma 2.5, the definition of $\Psi\left(\theta^{\iota-1} \rho\right)$ and (I) , for $\iota=1,2, \ldots, j-1$.

Since $\left|D l_{\theta^{j} \rho}\right| \leq 2 M$. We are in a position to apply Theorem 3.1. We obtain

$$
\begin{aligned}
\Phi\left(\theta^{j} \rho / 2,(D u)_{\theta^{j} \rho} / 2\right) & \leq \Phi\left(\theta^{j} \rho,(D u)_{\theta^{j} \rho}\right) \\
& \leq C_{\mathrm{cac}}(2 M) \tilde{\Psi}\left(\theta^{j} \rho\right) \\
& \leq C_{\mathrm{cac}}(2 M)\left(\theta^{2 \alpha j} \tilde{\Psi}(\rho)+C_{7}(2 M)\left(\theta^{j} \rho\right)^{2 \beta}\right) .
\end{aligned}
$$

We now consider $0<r \leq \rho / 2$. We fix $k \in N \cup\{0\}$ with $\theta^{k+1} \rho / 2<r \leq \theta^{k} \rho / 2$. Then the previous estimate implies

$$
\begin{aligned}
\Phi\left(r,(D u)_{r}\right) & \theta^{-n-2}\left[\left(1+|D l|^{2}\right)^{\frac{m-2}{2}} f_{Q_{\theta^{k} \rho / 2}\left(z_{0}\right)}\left|D u-(D u)_{\theta^{k} \rho / 2}\right|^{2} d z\right. \\
& \left.+f_{Q_{\theta^{k} \rho / 2}\left(z_{0}\right)}\left|D u-(D u)_{\theta^{k} \rho / 2}\right|^{m} d z\right] \\
\leq & \theta^{-n-2} C_{\mathrm{cac}}(2 M)\left[\theta^{2 \alpha k} \tilde{\Psi}(\rho)+C_{7}(M)\left(\theta^{k} \rho\right)^{2 \beta}\right] \\
\leq & \theta^{-n-2} C_{\mathrm{cac}}(2 M)\left[\theta^{-2 \alpha}\left(\frac{r}{\rho / 2}\right)^{2 \alpha} \tilde{\Psi}(\rho)+C_{7}(M) 2^{2 \beta} \theta^{-2 \beta} r^{2 \beta}\right] \\
\leq & 4 \theta^{-n-2-2 \alpha} C_{\mathrm{cac}}(2 M)\left[\left(\frac{r}{\rho / 2}\right)^{2 \alpha} \tilde{\Psi}(\rho)+\left(C_{7}(M)+1\right) r^{2 \beta}\right] \\
\leq & C_{\mathrm{dec}}(M)\left[\left(\frac{r}{\rho / 2}\right)^{2 \alpha} \tilde{\Psi}(\rho)+r^{2 \beta}\right] .
\end{aligned}
$$

Next, we show that $\left((D u)_{\theta^{j} \rho / 2}\right)_{j \in N}$ is a Cauchy sequence in $R^{n N}$. For $K>j$ we deduce

$$
\begin{aligned}
\left|(D u)_{\theta^{j} \rho / 2}-(D u)_{\theta^{k} \rho / 2}\right| & \leq \sum_{\iota=j+1}^{k}\left|(D u)_{\theta^{\imath} \rho / 2}-(D u)_{\theta^{\iota-1} \rho / 2}\right| \\
& \leq \sqrt{\theta^{-n-2}} \sum_{\imath=j}^{k-1}\left[f_{\theta^{\imath} \rho / 2\left(z_{0}\right)}\left|D u-(D u)_{\theta^{\iota} \rho / 2}\right|^{2} d z\right]^{\frac{1}{2}} \\
& =\sqrt{\theta^{-n-2}} \sum_{\imath=j}^{k-1} \sqrt{\Phi\left(\theta^{\iota} \rho / 2\right)}
\end{aligned}
$$




$$
\begin{aligned}
& \leq \sqrt{\theta^{-n-2} C_{\mathrm{cac}}(2 M)} \sum_{\imath=j}^{k-1} \sqrt{\theta^{2 \alpha \iota} \tilde{\Psi}(\rho)+C_{7}(M)\left(\theta^{\iota} \rho\right)^{2 \beta}} \\
& \leq \sqrt{\theta^{-n-2} C_{\mathrm{cac}}(2 M)} \sum_{\imath=j}^{k-1}\left(\frac{\sqrt{\tilde{\Psi}(\rho)}}{1-\theta^{\alpha}} \theta^{\alpha j}+\frac{\sqrt{C_{7}(M) \rho^{2 \beta}}}{1-\theta^{\beta}}\right) .
\end{aligned}
$$

This proves the claim. Therefore the limit $\Gamma_{z_{0}}=\lim _{j \rightarrow \infty}(D u)_{\theta^{j} \rho / 2} \in R^{n N}$ exists and from the previous estimate, we infer (taking the limit $k \rightarrow \infty$ )

$$
\left|(D u)_{\theta^{j} \rho / 2}-\Gamma_{z_{0}}\right| \leq C_{8}(M) \sqrt{\theta^{2 \alpha j} \tilde{\Psi}(\rho)+\left(\theta^{j} \rho\right)^{2 \beta}} .
$$

Combining this with (4.20), we arrive at

$$
\begin{aligned}
(1 & \left.+\left|\Gamma_{z_{0}}\right|^{2}\right)^{\frac{m-2}{2}} f_{\theta^{j} \rho / 2}\left|D u-\Gamma_{z_{0}}\right|^{2} d z+f_{\theta^{j} \rho / 2}\left|D u-\Gamma_{z_{0}}\right|^{m} d z \\
& \leq 2^{m-1} \Phi\left(\theta^{j} \rho / 2\right)+2\left|(D u)_{\theta^{j} \rho / 2}-\Gamma_{z_{0}}\right|^{2}+2^{m-1}\left|(D u)_{\theta^{j} \rho / 2}-\Gamma_{z_{0}}\right|^{m} \\
& \leq C(M)\left(\theta^{2 \alpha j} \tilde{\Psi}(\rho)+\rho^{2 \beta}\right) .
\end{aligned}
$$

For $0<r \leq \rho / 2$, we find $k \in N \cup\{0\}$ with $\theta^{k+1} \rho / 2<r<\theta^{k} \rho / 2$. Then the previous estimate implies

$$
\begin{aligned}
& \left(1+\left|\Gamma_{z_{0}}\right|^{2}\right)^{\frac{m-2}{2}} f_{Q_{r}\left(z_{0}\right)}\left|D u-\Gamma_{z_{0}}\right|^{2} d z+f_{Q_{r}\left(z_{0}\right)}\left|D u-\Gamma_{z_{0}}\right|^{m} d z \\
& \quad \leq \theta^{-n-2}\left[\left(1+\left|\Gamma_{z_{0}}\right|^{2}\right)^{\frac{m-2}{2}} f_{\theta^{k} \rho / 2}\left|D u-\Gamma_{z_{0}}\right|^{2} d z+f_{\theta^{k} \rho / 2}\left|D u-\Gamma_{z_{0}}\right|^{m} d z\right] \\
& \quad \leq \theta^{-n-2} C(M)\left[\theta^{2 \alpha k} \tilde{\Psi}(\rho)+\left(\theta^{k} \rho\right)^{2 \beta}\right] \\
& \quad \leq C(M)\left[\left(\frac{r}{\rho / 2}\right)^{2 \alpha} \Psi(\rho)+r^{2 \beta}\right] .
\end{aligned}
$$

This proves the assertion of the lemma.

An immediate consequence of the previous lemma and of isomorphism theorem of Campanato-Da Prato [16] is the following result.

Theorem 4.1 (Description of regularity points) Let $u \in L^{m}\left(-T, 0 ; W^{1, m}\left(\Omega, R^{N}\right)\right) \cap L^{\infty}\left(Q_{T}\right.$; $\left.R^{N}\right)$ be a weak solution to the system (1.9) under the assumptions (H1)-(H3) and (H4'), (H5), and denote by $\Sigma$ the singular set of $u$. Then $\Sigma \subset \Sigma_{0} \cup \Sigma_{2}$, where

$$
\begin{aligned}
\Sigma_{0}= & \left\{z_{0} \in Q_{T}: \lim \inf _{\rho \rightarrow 0} \rho^{-m} f_{Q_{\rho}\left(z_{0}\right)}\left|u-(u)_{z_{0}, \rho}-(D u)_{z_{0}, \rho}\left(x-x_{0}\right)\right|^{m} d z>0\right\} \\
& \cup\left\{z_{0} \in Q_{T}: \lim \inf _{\rho \rightarrow 0} f_{Q_{\rho}\left(z_{0}\right)}\left|D u-(D u)_{z_{0}, \rho}\right|^{m} d z>0\right\},
\end{aligned}
$$

and

$$
\Sigma_{2}=\left\{z_{0} \in Q_{T}: \lim \sup _{\rho \rightarrow 0}\left(\left|(u)_{z_{0}, \rho}\right|+\left|(D u)_{z_{0}, \rho}\right|\right)=\infty\right\} .
$$


At last, we have the following.

Theorem 4.2 (Almost everywhere regularity) Let $u \in L^{m}\left(-T, 0 ; W^{1, m}\left(\Omega, R^{N}\right)\right) \cap L^{\infty}\left(Q_{T}\right.$; $\left.R^{N}\right)$ be a weak solution to the system (1.9) under the assumptions (H1)-(H3) and (H4), (H5), and denote by $\Sigma$ the singular set of $u$. Then $\Sigma \subset \Sigma_{1} \cup \Sigma_{2}$, where $\Sigma_{2}$ is as in Theorem 4.1 and

$$
\begin{aligned}
\Sigma_{1}= & \left\{z_{0} \in Q_{T}: \lim \inf _{\rho \rightarrow 0} f_{Q_{\rho}\left(z_{0}\right)}\left|D u-(D u)_{z_{0}, \rho}\right|^{m} d z>0\right\} \\
& \cup\left\{z_{0} \in Q_{T}: \lim \inf _{\rho \rightarrow 0} f_{Q_{\rho}\left(z_{0}\right)}\left|u-(u)_{z_{0}, \rho}\right|^{m} d z>0\right\} .
\end{aligned}
$$

Proof We start taking a point $z_{0}\left(x_{0}, t_{0}\right) \in Q_{T}$ such that

$$
\begin{aligned}
& \lim \inf _{\rho \rightarrow 0} f_{Q_{\rho}\left(z_{0}\right)}\left|D u-(D u)_{z_{0}, \rho}\right|^{m} d z=0, \\
& \lim \inf _{\rho \rightarrow 0} f_{Q_{\rho}\left(z_{0}\right)}\left|u-(u)_{z_{0}, \rho}\right|^{m} d z=0,
\end{aligned}
$$

and

$$
\sup _{\rho>0}\left|(u)_{z_{0}, \rho}\right|+\sup _{\rho>0}\left|(D u)_{z_{0}, \rho}\right| \leq M<\infty
$$

The proof is complete if we show that such points are regularity points.

Step 1: a comparison estimate. Consider the unique weak solution $v \in L^{m}\left(t_{0}-4 \rho^{2}, t_{0}\right.$; $\left.W^{1, m}\left(B_{\rho}\left(x_{0}\right), R^{N}\right)\right)$ of the initial boundary value problem

$$
\left\{\begin{array}{l}
\int_{Q_{2 \rho}\left(z_{0}\right)}\left(v^{i} \varphi_{t}^{i}-A_{i}^{\alpha}\left(z_{0},(u)_{z_{0}, \rho}, D v\right) D_{\alpha} \varphi^{i}\right) d z=0, \quad \forall \varphi \in C_{0}^{\infty}\left(Q_{2 \rho}\left(z_{0}\right), R^{N}\right) \\
v=u, \quad \text { on } B_{2 \rho\left(x_{0}\right)} \times\left\{t_{0}-4 \rho^{2}\right\} \cap \partial B_{2 \rho}\left(x_{0}\right) \times\left(t_{0}-4 \rho^{2}, t_{0}\right) .
\end{array}\right.
$$

Then the difference $u-v$ satisfies

$$
\begin{aligned}
& \int_{Q_{2 \rho}\left(z_{0}\right)}\left[(u-v)^{i} \varphi_{t}^{i}-\left(A_{i}^{\alpha}(z, u, D u)-A_{i}^{\alpha}\left(z_{0},(u)_{z_{0}, \rho}, D v\right)\right) D_{\alpha} \varphi^{i}\right] d z \\
& \quad+\int_{Q_{2 \rho}\left(z_{0}\right)} B_{i}(z, u, D u) \varphi^{i} d z=0
\end{aligned}
$$

for every $\varphi \in C_{0}^{\infty}\left(Q_{2 \rho}\left(z_{0}\right), R^{N}\right)$. We now choose $\varphi=\chi(t)(u-v)^{i}$ with $\chi \equiv 1$ for $(-\infty, s)$, $\chi \equiv 0$ on $(s+\varepsilon, \infty)$, and $\chi(t)=(s+\varepsilon-t) / \varepsilon$ for $s \leq t \leq s+\varepsilon$, where $[s, s+\varepsilon] \in\left(t_{0}-4 \rho^{2}, t_{0}\right)$.

Then

$$
\begin{aligned}
& \frac{1}{2} \int_{Q_{2 \rho}\left(z_{0}\right)} \partial_{t}\left(|u-v|^{2} \chi\right) d z+\frac{1}{2} \int_{Q_{2 \rho}\left(z_{0}\right)}|u-v|^{2} \partial_{t} \chi d z \\
& \quad-\int_{Q_{2 \rho}\left(z_{0}\right)}\left(A_{i}^{\alpha}(z, u, D u)-A_{i}^{\alpha}\left(z_{0},(u)_{z_{0}, \rho}, D v\right)\right)\left(D u^{i}-D v^{i}\right) \chi d z \\
& \quad+\int_{Q_{2 \rho}\left(z_{0}\right)} B_{i}(z, u, D u) \chi(t)(u-v)^{i} d z=0 .
\end{aligned}
$$


Letting $\varepsilon \rightarrow 0$, we easily obtain that for a.e. $s \in\left(t_{0}-4 \rho^{2}, t_{0}\right)$

$$
\begin{aligned}
& \frac{1}{2}\|u(\cdot, s)-v(\cdot, s)\|_{L^{2}\left(B_{2 \rho}\left(x_{0}\right)\right)}^{2} \\
& \quad+\int_{B_{2 \rho}\left(x_{0}\right) \times\left(t_{0}-4 \rho^{2}, t_{0}\right)}\left(A_{i}^{\alpha}\left(z_{0},(u)_{z_{0}, \rho}, D u\right)-A_{i}^{\alpha}\left(z_{0},(u)_{z_{0}, \rho}, D v\right)\right) D(u-v)^{i} \chi d z \\
& =\int_{B_{2 \rho}\left(x_{0}\right) \times\left(t_{0}-4 \rho^{2}, s\right)}\left(A_{i}^{\alpha}\left(z_{0},(u)_{z_{0}, \rho}, D u\right)-A_{i}^{\alpha}(z, u, D u)\right) D(u-v)^{i} d z \\
& \quad+\int_{B_{2 \rho}\left(x_{0}\right) \times\left(t_{0}-4 \rho^{2}, s\right)} B_{i}(z, u, D u)(u-v)^{i} d z \\
& =0 .
\end{aligned}
$$

The second term of the left-hand side of the previous equation can be estimated by the use of monotonicity, i.e., (H3). We therefore obtain

$$
\begin{aligned}
& \frac{1}{2}\|u(\cdot, s)-v(\cdot, s)\|_{L^{2}\left(B_{2 \rho}\left(x_{0}\right)\right)}^{2}+\lambda\left(1+|D v|^{2}+|D u|^{2}\right)^{\frac{m-2}{2}} \int_{B_{2 \rho}\left(x_{0}\right) \times\left(t_{0}-4 \rho^{2}, s\right)}|D u-D v|^{2} d z \\
& \leq \int_{B_{2 \rho}\left(x_{0}\right) \times\left(t_{0}-4 \rho^{2}, s\right)}\left(A_{i}^{\alpha}\left(z_{0},(u)_{z_{0}, \rho}, D u\right)-A_{i}^{\alpha}(z, u, D u)\right) D(u-v)^{i} d z \\
& \quad+\int_{B_{2 \rho}\left(x_{0}\right) \times\left(t_{0}-4 \rho^{2}, s\right)} B_{i}(z, u, D u)(u-v)^{i} d z \\
& =I+I I .
\end{aligned}
$$

To estimate the right-hand side, we use (H4) which easily yields

$$
\begin{aligned}
& \left|A_{i}^{\alpha}\left(z_{0},(u)_{z_{0}, \rho}, D u\right)-A_{i}^{\alpha}(z, u, D u)\right| \\
& \quad \leq L \theta\left(2\left|(u)_{z_{0}, 2 \rho}\right|+\left|u-(u)_{z_{0}, 2 \rho}\right|, 4 \rho+\left|u-(u)_{z_{0}, 2 \rho}\right|\right)(1+|D u|)^{\frac{m}{2}} .
\end{aligned}
$$

Using the previous estimate, Young's inequality and the fact that $\theta \leq 1$, we have

$$
\begin{aligned}
|I| \leq & \frac{\lambda}{2}\left(1+|D v|^{2}+|D u|^{2}\right)^{\frac{m-2}{2}} \int_{B_{2 \rho}\left(x_{0}\right) \times\left(t_{0}-4 \rho^{2}, s\right)}|D u-D v|^{2} d z \\
& +\frac{2 L^{2}}{\lambda} \int_{Q_{2 \rho}\left(z_{0}\right)} \theta\left(2\left|(u)_{z_{0}, 2 \rho}\right|+\left|u-(u)_{z_{0}, 2 \rho}\right|, 4 \rho+\left|u-(u)_{z_{0}, 2 \rho}\right|\right)\left(1+|D u|^{m}\right) d z .
\end{aligned}
$$

Having combined the previous estimate with (4.23), we arrive at

$$
\begin{aligned}
& \frac{1}{2}\|u(\cdot, s)-v(\cdot, s)\|_{L^{2}\left(B_{2 \rho}\left(x_{0}\right)\right)}^{2}+\frac{\lambda}{2}\left(1+|D v|^{2}+|D u|^{2}\right)^{\frac{m-2}{2}} \int_{B_{2 \rho}\left(x_{0}\right) \times\left(t_{0}-4 \rho^{2}, s\right)}|D u-D v|^{2} d z \\
& \quad \leq \frac{2 L^{2}}{\lambda} \int_{Q_{2 \rho}\left(z_{0}\right)} \theta\left(2\left|(u)_{z_{0}, 2 \rho}\right|+\left|u-(u)_{z_{0}, 2 \rho}\right|, 4 \rho+\left|(u)_{z_{0}, 2 \rho}\right|\right)\left(1+|D u|^{m}\right) d z+I I \\
& \quad=\frac{2 L^{2}}{\lambda} I I I+I I .
\end{aligned}
$$

We shall provide on estimate for III. We denote $\varepsilon_{\sigma}=f_{Q_{z_{0}}(\sigma)}\left|u-(u)_{z_{0}, \sigma}\right| d z, \sigma>0$. 
If we let $A_{t}^{\sigma} \equiv A_{t}=\left\{z \in Q_{2 \rho}\left(z_{0}\right):\left|u-(u)_{z_{0}, 2 \rho}\right| \geq t\right\}$, then

$$
\left|A_{t}\right| \leq \frac{1}{t} \int_{Q_{2 \rho}\left(z_{0}\right)}\left|u-(u)_{z_{0}, 2 \rho}\right| d z \leq \frac{\left|Q_{2 \rho}\right|}{t} \varepsilon_{2 \rho} .
$$

We now split III

$$
I I I=\int_{A_{t}}(\cdots) d z+\int_{Q_{2 \rho}\left(z_{0}\right) \backslash A_{t}}(\cdots) d z=I V+V
$$

and estimate $I V$ and $V$. We have, using that $\theta \leq 1,(4.25)$ and (4.22)

$$
\begin{aligned}
I V & \leq 2^{m-1} \int_{Q_{2 \rho}\left(z_{0}\right)}\left|D u-(D u)_{z_{0}, 2 \rho}\right|^{m} d z+\left(1+2^{m-1}\left|(D u)_{z_{0}, 2 \rho}\right|^{m}\right)\left|A_{t}\right| \\
& \leq 2^{m-1} \int_{Q_{2 \rho}\left(z_{0}\right)}\left|D u-(D u)_{z_{0}, 2 \rho}\right|^{m} d z+2^{m-1}\left(1+2 M^{2}\right) \frac{\left|Q_{2 \rho}\right|}{t} \varepsilon_{2 \rho} .
\end{aligned}
$$

From the definition of $\theta$, we have

$$
V \leq 4 K(2 M+t)(\rho+t)^{\beta} \int_{Q_{2 \rho}\left(z_{0}\right)}\left(1+|D u|^{m}\right) d z .
$$

Noting that $\sup _{Q_{T}} u=V$, we have

$$
\begin{aligned}
I I \leq & 2^{m-1} a V \int_{Q_{2 \rho}\left(z_{0}\right)}\left|D u-(D u)_{z_{0}, 2 \rho}\right|^{m} d z+\int_{Q_{2 \rho}\left(z_{0}\right)} \frac{|u-v|^{2}}{\rho^{2}} d z \\
& +\left(2^{m-1} a M^{m}+b\right) \alpha_{n} \rho^{n+2} \rho^{2} .
\end{aligned}
$$

We now choose the parameter $t$ carefully, i.e., $t=\sqrt{\varepsilon_{2 \rho}}$ and let $\varepsilon$ suitably small. Then connecting the previous estimates for $I I, I I I, I V$ and $V$ to (4.24), we easily have the estimate we were interested in, that is,

$$
\begin{aligned}
& \left(1+|D v|^{2}\right)^{\frac{m-2}{2}} f_{Q_{2 \rho}\left(z_{0}\right)}|D u-D v|^{2} d z+f_{Q_{2 \rho}\left(z_{0}\right)}|D u-D v|^{m} d z \\
& \quad+\sup _{t_{0}-4 \rho^{2} \leq t \leq t_{0}} \rho^{-2} f_{B_{2 \rho}\left(x_{0}\right)}|u(x, t)-v(x, t)|^{2} d z \\
& \leq C\left[K\left(2 M+\sqrt{\varepsilon_{2 \rho}}\right)\left(\rho+\sqrt{\varepsilon_{2 \rho}}\right)^{\beta}+1\right] f_{Q_{2 \rho}\left(z_{0}\right)}\left|D u-(D u)_{z_{0}, 2 \rho}\right|^{m} d z \\
& \quad+C\left[K\left(2 M+\sqrt{\varepsilon_{2 \rho}}\right)\left(\rho+\sqrt{\varepsilon_{2 \rho}}\right)^{\beta}+\sqrt{\varepsilon_{2 \rho}}\right]\left(1+M^{m}\right)+C \rho^{2}\left(a M^{m}+b\right) \\
& =S(\rho) .
\end{aligned}
$$

In particular, we see that

$$
\begin{aligned}
\rho^{-2} f_{Q_{2 \rho}\left(z_{0}\right)}|u(z)-v(z)|^{2} d z & \leq \sup _{t_{0}-4 \rho^{2} \leq t \leq t_{0}} \rho^{-2} f_{B_{2 \rho}\left(x_{0}\right)}|u(x, t)-v(x, t)|^{2} d x \\
& \leq S(\rho) .
\end{aligned}
$$


We observe that, as a consequence of (4.21) and (4.22), we have that

$$
\lim \inf _{\rho \mapsto 0} S(\rho)=0 .
$$

Step 2: A Poincare-type inequality. Let us define

$$
\tilde{v}=v-(D u)_{z_{0}, 2 \rho}\left(x-x_{0}\right) .
$$

Therefore $\tilde{v}$ solves

$$
\int_{Q_{\rho}\left(z_{0}\right)}\left(\tilde{v}^{i} \varphi_{t}^{i}-\tilde{A}_{i}^{\alpha}(D \tilde{v}) D_{\alpha} \varphi^{i}\right) d z=0, \quad \forall \varphi \in C_{0}^{\infty}\left(Q_{2 \rho}\left(z_{0}\right), R^{N}\right),
$$

where $\tilde{A}_{i}^{\alpha}(p)=A_{i}^{\alpha}\left(z_{0},(u)_{z_{0}, 2 \rho},(D v)_{z_{0}, 2 \rho}+p\right)$ for every $p \in R^{n N}$. From [17], Theorem 3.1, we conclude that $\tilde{v} \in W^{1,2}\left(t_{0}-\rho^{2}, t_{0} ; W^{1,2}\left(B_{2 \rho\left(x_{0}\right)}, R^{N}\right)\right)$ and that

$$
\begin{aligned}
\rho^{2} f_{Q_{\rho}\left(z_{0}\right)}\left|\partial_{t} v\right|^{2} d z & =\rho^{2} f_{Q_{\rho}\left(z_{0}\right)}\left|\partial_{t} \tilde{v}\right|^{2} d z \leq C(\lambda, L) f_{Q_{2 \rho}\left(z_{0}\right)}|D \tilde{v}|^{2} d z \\
& =C(\lambda, L) f_{Q_{2 \rho}\left(z_{0}\right)}\left|D v-(D v)_{z_{0}, 2 \rho}\right|^{2} d z \\
& \leq C(\lambda, L)\left[f_{Q_{2 \rho}\left(z_{0}\right)}|D u-D v|^{2} d z+f_{Q_{2 \rho}\left(z_{0}\right)}\left|D u-(D v)_{z_{0}, 2 \rho}\right|^{2} d z\right] .
\end{aligned}
$$

In view of the previous estimate, using the Poincare inequality for $v$ and (4.26), we find

$$
\begin{aligned}
& \rho^{-m} f_{Q_{\rho}\left(z_{0}\right)}\left|v-(v)_{z_{0}, \rho}-(D v)_{z_{0}, \rho}\left(x-x_{0}\right)\right|^{m} d z \\
& \quad \leq C\left[f_{Q_{\rho}\left(z_{0}\right)}\left|D v-(D v)_{z_{0}, \rho}\right|^{m} d z+\rho^{m} f_{Q_{\rho}\left(z_{0}\right)}\left|\partial_{t} v\right|^{m} d z\right] \\
& \quad \leq C\left[f_{Q_{2 \rho}\left(z_{0}\right)}|D u-D v|^{m} d z+f_{Q_{\rho}\left(z_{0}\right)}\left|D u-(D u)_{z_{0}, 2 \rho}\right|^{m} d z\right] \\
& \quad \leq C\left[S(\rho)+f_{Q_{\rho}\left(z_{0}\right)}\left|D u-(D u)_{z_{0}, 2 \rho}\right|^{m} d z\right],
\end{aligned}
$$

where $C=C(n, \lambda, L)$.

Finally, by comparison, we get the Poincare inequality for $u$ via (4.26) and the previous estimate

$$
\begin{aligned}
\rho^{-m} & f_{Q_{\rho}\left(z_{0}\right)}\left|u-(u)_{z_{0}, \rho}-(D u)_{z_{0}, \rho}\left(x-x_{0}\right)\right|^{m} d z \\
\leq & C\left[\rho^{-m} f_{Q_{\rho}\left(z_{0}\right)}|u-v|^{m} d z+f_{Q_{\rho}\left(z_{0}\right)}|D u-D v|^{m} d z\right. \\
& \left.+\rho^{-m} f_{Q_{\rho}\left(z_{0}\right)}\left|v-(v)_{z_{0}, \rho}-(D u)_{z_{0}, \rho}\left(x-x_{0}\right)\right|^{m} d z\right] \\
\leq & C\left[S(\rho)+f_{Q_{2 \rho}\left(z_{0}\right)}\left|D u-(D u)_{z_{0}, 2 \rho}\right|^{m} d z\right]
\end{aligned}
$$

for a constant $C=C(n, \lambda, L)$. 
Step 3: Conclusion. From the previous estimate and (4.28), the assertion readily follows. Indeed if $z_{0} \in Q_{T}$ satisfies (4.21) and (4.22), then we have

$$
\lim \inf _{\rho \rightarrow 0} \rho^{-m} f_{Q_{\rho}\left(z_{0}\right)}\left|u-(u)_{z_{0}, \rho}-(D u)_{z_{0}, \rho}\left(x-x_{0}\right)\right|^{m} d z=0
$$

therefore $z_{0}$ is a regular point in view of Theorem 4.1 .

\section{Competing interests}

The authors declare that they have no competing interests.

\section{Authors' contributions}

SC participated in design of the study and drafted the manuscript. ZT participated in conceived of the study and the amendment of the paper. All authors read and approved the final manuscript.

\section{Author details}

${ }^{1}$ School of Mathematics and Statistics, Minnan Normal University, Zhangzhou, Fujian 363000, China. ${ }^{2}$ School of Mathematical Science, Xiamen University, Xiamen, Fujian 361005, China.

\section{Acknowledgements}

Supported by the National Natural Science Foundation of China (Nos: 11201415, 11271305), the Natural Science Foundation of Fujian Province (2012J01027) and the Training Programme Foundation for Excellent Youth Researching Talents of Fujian's Universities (JA12205).

Received: 17 August 2012 Accepted: 10 May 2013 Published: 26 June 2013

\section{References}

1. Wittle, M: Computer simulations of an electrorheological fluid. J. Non-Newton. Fluid Mech. 37, $233-263$ (1993)

2. Rajagopal, KR, Ruzicka, M: On the modeling of electrorheological materials. Mech. Res. Commun. 23, 401-407 (1996)

3. Rajagopal, KR, Ruzicka, M: Mathematical modeling of electrorheological materials. Contin. Mech. Thermodyn. 13, 59-78 (2001)

4. Duzaar, F, Mingione, G: Second order parabolic systems, optimal regularity, and singular sets of solutions. Ann. Inst. Henri Poincaré, Anal. Non Linéaire 22, 705-751 (2005)

5. Duzaar, F, Grotowski, JF: Optimal interior partial regularity for nonlinear elliptic systems: the method of $A$-harmonic approximation. Manuscr. Math. 103, 267-298 (2000)

6. Chen, $\mathrm{S}, \mathrm{Tan}, \mathrm{Z}$ : The method of $p$-harmonic approximation and optimal interior partial regularity for energy minimizing $p$-harmonic maps under the controllable growth condition. Sci. China Ser. A 50(1), 105-115 (2007)

7. Chen, S, Tan, Z: The method of A-harmonic approximation and optimal interior partial regularity for nonlinear elliptic systems under the controllable growth condition. J. Math. Anal. Appl. 335, 20-42 (2007)

8. Chen, S, Tan, Z: Optimal interior partial regularity for nonlinear elliptic systems. Discrete Contin. Dyn. Syst., Ser. A 27(3), 981-993 (2010)

9. Chen, S, Tan, Z: Optimal interior partial regularity for nonlinear elliptic systems for the case $1<m<2$ under natural growth condition. J. Inequal. Appl. 2010, Article ID 680714 (2010)

10. Zhong, T: $C^{1, \alpha}$-partial regularity of nonlinear parabolic systems. J. Partial Differ. Equ. 5, 23-34 (1992)

11. De Giorgi, E: Frontiere orientate di misura minima, Sem. Scuola Normale Superiore Pisa (1960-1961)

12. De Giorgi, E: Un esempio di estremali discontinue per un problema variazionale di tipo ellittico. Boll. Unione Mat. Ital. 1(4), 135-137 (1968)

13. Simon, L: Compact sets in the space $L^{P}(0, T ; B)$. Ann. Mat. Pura Appl. 146(4), 65-96 (1987)

14. Kronz, M: Partial regularity results for minimizers of quasiconvex functionals of higher order. Ann. Inst. Henri Poincaré, Anal. Non Linéaire 19, 81-112 (2002)

15. Campanato, S: Equazioni paraboliche del secondo ordine e spazi $\mathcal{L}^{2, \theta}(\Omega, \delta)$. Ann. Mat. Pura Appl. 73(4), 55-102 (1996)

16. Da Prato, G: Spazi $\mathcal{L}^{2, \theta}(\Omega, \delta)$ e loro proprieta. Ann. Mat. Pura Appl. 69(4), 383-392 (1965)

17. Campanato, S: On the nonlinear parabolic systems in divergence form Hölder continuity and partial Hölder continuity of the solutions. Ann. Mat. Pura Appl. 137(4), 83-122 (1984) 